\title{
Identification of miRNAs as the Crosstalk in the Interaction between Neural Stem/Progenitor Cells and Endothelial Cells
}

\author{
Xin Wang $\left(\mathbb{D},{ }^{1}\right.$ Simin Li $\left(\mathbb{D},{ }^{2}\right.$ Yihong Ma $\left(\mathbb{D},{ }^{3}\right.$ Yuzhen Xu $\mathbb{( D},{ }^{4}$ \\ Anthony Chukwunonso Ogbuehi $\mathbb{D}^{5},{ }^{2}$ Xianda Hu $\mathbb{D}^{6},{ }^{6}$ Aneesha Acharya ${ }^{(D)},{ }^{7}$ Rainer Haak ${ }^{D},{ }^{2}$ \\ Dirk Ziebolz $\mathbb{D},{ }^{2}$ Gerhard Schmalz $\mathbb{D}^{2}{ }^{2}$ Hanluo Li $\mathbb{D}^{8},{ }^{8}$ Sebastian Gaus $\mathbb{D}^{8},{ }^{8}$ Bernd Lethaus $\mathbb{D}^{8},{ }^{8}$ \\ Vuk Savkovic $\mathbb{D}^{8}{ }^{8}$ and Zhiqiang Su $\mathbb{D D}^{1}$ \\ ${ }^{1}$ Department of Neurology, First Affiliated Hospital of Harbin Medical University, Harbin 150001, China \\ ${ }^{2}$ Department of Cariology, Endodontology and Periodontology, University Leipzig, Liebigstr. 12, 04103 Leipzig, Germany \\ ${ }^{3}$ Department of Neurology, Graduate School of Medical Sciences, Faculty of Life Sciences, Kumamoto University, Kumamoto, Japan \\ ${ }^{4}$ Department of Neurology, Shanghai Tenth People's Hospital, Tongji University School of Medicine, No. 301 Middle Yanchang Road, \\ Shanghai, China \\ ${ }^{5}$ Faculty of Physics, University of Münster, Wilhelm-Klemm-Straße 9, 48149 Münster, Germany \\ ${ }^{6}$ Laboratory of Molecular Cell Biology, Beijing Tibetan Hospital, China Tibetology Research Center, 218 Anwaixiaoguanbeili Street, \\ Chaoyang, Beijing 100029, China \\ ${ }^{7}$ Faculty of Dentistry, University of Hong Kong, Hong Kong, China \\ ${ }^{8}$ Department of Cranio Maxillofacial Surgery, University Clinic Leipzig, Liebigstr. 12, 04103 Leipzig, Germany
}

Correspondence should be addressed to Zhiqiang Su; suzhiqiang2020@126.com

Received 9 October 2020; Revised 22 November 2020; Accepted 24 November 2020; Published 16 December 2020

Academic Editor: Wen-Jun Tu

Copyright (c) 2020 Xin Wang et al. This is an open access article distributed under the Creative Commons Attribution License, which permits unrestricted use, distribution, and reproduction in any medium, provided the original work is properly cited.

\begin{abstract}
Aim. This study is aimed at identifying genetic and epigenetic crosstalk molecules and their target drugs involved in the interaction between neural stem/progenitor cells (NSPCs) and endothelial cells (ECs). Materials and Methods. Datasets pertaining to reciprocal mRNA and noncoding RNA changes induced by the interaction between NSPCs and ECs were obtained from the GEO database. Differential expression analysis (DEA) was applied to identify NSPC-induced EC alterations by comparing the expression profiles between monoculture of ECs and ECs grown in EC/NSPC cocultures. DEA was also utilized to identify EC-induced NSPC alterations by comparing the expression profiles between monoculture of NSPCs and NSPCs grown in EC/NSPC cocultures. The DEGs and DEmiRNAs shared by NSPC-induced EC alterations and EC-induced NSPC alterations were then identified. Furthermore, miRNA crosstalk analysis and functional enrichment analysis were performed, and the relationship between DEmiRNAs and small molecular drug targets/environment chemical compounds was investigated. Results. One dataset (GSE29759) was included and analyzed in this study. Six genes (i.e., MMP14, TIMP3, LOXL1, CCK, SMAD6, and HSPA2), three miRNAs (i.e., miR-210, miR-230a, and miR-23b), and three pathways (i.e., Akt, ERK1/2, and BMPs) were identified as crosstalk molecules. Six small molecular drugs (i.e., deptropine, fluphenazine, lycorine, quinostatin, resveratrol, and thiamazole) and seven environmental chemical compounds (i.e., folic acid, dexamethasone, choline, doxorubicin, thalidomide, bisphenol A, and titanium dioxide) were identified to be potential target drugs of the identified DEmiRNAs. Conclusion. To conclude, three miRNAs (i.e., miR-210, miR-230a, and miR-23b) were identified to be crosstalks linking the interaction between ECs and NSPCs by implicating in both angiogenesis and neurogenesis. These crosstalk molecules might provide a basis for devising novel strategies for fabricating neurovascular models in stem cell tissue engineering.
\end{abstract}




\section{Introduction}

It is well known that the neurovascular unit (NVU) comprises a collection of cells (e.g., endothelial, neural, and glial cells), which can control interactions between neurons and the vasculature. Based on the concept of NVU, the construction of 3D neurovascular tissue has emerged as a promising approach in the field of tissue engineering $[1,2]$. Constructing $3 \mathrm{D}$ neurovascular tissues by combining neurogenesis and angiogenesis models is viewed as a potentially effective strategy to facilitate functional recovery in ischemic stroke [3]. Increasing evidence has shown that the cell contactdependent interactions between neural stem/progenitor cells (NSPCs) and endothelial cells (ECs) can drive the coupling of neurogenesis and angiogenesis. On the one hand, NSPCs can promote morphogenesis and angiogenesis of ECs by expressing angiogenic factors such as vascular endothelial growth factor (VEGF) [4]. In turn, VEGF has been demonstrated to promote neurogenesis, neuronal patterning, neuroprotection, and glial growth of NSPCs [5]. On the other hand, ECs can also stimulate survival, proliferation, neuronal differentiation, and neurogenesis of NSPCs by secreting neurotrophic factors such as brain-derived neurotrophic factor (BDNF) [6-8]. Conversely, BDNF has been shown to support the cell-cell contacts and survival of ECs, suggesting that it plays a critical role in maintaining vessel stabilization and mediating angiogenesis [9]. Overall, current evidence indicates that the interactions between NSPCs and ECs are governed by several common crosstalk factors that regulate both the neurogenic and angiogenic processes.

A previous study using microarray analysis investigated the molecular mechanisms underlying the interaction between NSPCs and ECs at the mRNA and miRNA levels [10]. This study focused on investigating the NSPC-induced molecular alterations in the angiogenesis of ECs and the EC-induced molecular alterations in the neurogenesis of NSPCs. However, this study did not explore the genetic and epigenetic crosstalks underlying the reciprocal regulations between NSPCs and ECs from a systemic and comprehensive perspective. Bioinformatics techniques can be used to analyze and interpret data obtained by microarray datasets in order to identify the crosstalk between NSPCs and ECs at the gene and microRNA (miRNA) levels. Since targeting miRNAs with small molecules and environmental chemical compounds is a promising therapeutic strategy for human diseases $[11,12]$, it is necessary to identify the molecules and compounds that might be utilized in targeted drug delivery to construct neurovascular tissue. To the authors' knowledge, this is the first report using bioinformatics techniques to identify the crosstalk genes, signaling pathways, and miRNAs, as well as the crosstalk miRNA targeting drugs that are involved in the reciprocal regulation between NSPCs and ECs. As crosstalk molecules and their related drugs identified in this study are implicated in regulating neurogenic and angiogenic processes, their identification can potentially enable the development of novel strategies for fabricating neurovascular models using $3 \mathrm{D}$ tissue engineering.

\section{Materials and Methods}

2.1. Procurement of Microarray Datasets. A single dataset (GSE29759) using the Mus musculus model was retrieved from the Gene Expression Omnibus (GEO) database [10]. This dataset investigated both the mRNA and miRNA expression profiling. The study design of this dataset was established with four groups: monoculture of ECs (group A), ECs grown in EC/NSPC cocultures (group B), monoculture of NSPCs (group C), and NSPCs grown in EC/NSPC cocultures (group D).

These four groups were divided into two categories. First, the NSPC-induced EC alterations were analyzed by comparing expression profiles between groups $\mathrm{A}$ and $\mathrm{B}$. In the comparison between groups $A$ and B, group A was considered the control group, while group B was regarded as the experimental group. Second, the EC-induced NSPC changes were analyzed by comparing groups $\mathrm{C}$ and $\mathrm{D}$. Likewise, in the comparison between groups $\mathrm{C}$ and $\mathrm{D}$, group $\mathrm{C}$ was considered the control group, while group $\mathrm{D}$ was regarded as the experimental group.

2.2. Differential Expression Analysis. Differential expression analysis was performed using the R package "limma" [13] for identifying differentially expressed genes (DEGs) and differentially expressed miRNAs (DEmiRNAs) relevant to ECinduced NSPC changes and NSPC-induced EC changes, respectively. Genes and miRNAs with $P$ value $<0.05$ and $\mid$ $\log 2 \mathrm{FC} \mid \geq 0.5$ were considered DEGs and DEmiRNAs. Overlapping DEGs and DEmiRNAs between these two categories were determined using Venn diagrams.

2.3. Functional Enrichment Analysis of DEGs. Functional enrichment analysis of the overlapping DEGs was based on Gene Ontology (GO) terms, especially biological process (BP), as well as KEGG (Kyoto Encyclopedia of Genes and Genomes) pathways. This analysis was conducted by using the clusterProfiler package in the $\mathrm{R}$ program (significance level $P<0.05)[14]$.

2.4. The Construction of a PPI Network. Protein-protein interaction (PPI) pairs corresponding to DEGs were obtained using the STRING database [15]. Protein-protein interaction (PPI) networks of NSPC-induced EC changes (EC-PPI) and EC-induced NSPC changes (NSPC-PPI) were constructed with the "Cytoscape" software platform [16]. The topological characteristics of each PPI network were determined using the "NetworkAnalyzer" tool. A Venn diagram was used to identify the overlapping DEGs between the EC-PPI network and the NSPC-PPI network.

2.5. Identification of Transcription Factors-Target DEGs. To identify regulatory transcription factors (TFs) that control the DEGs at a transcriptional level, TF-target DEG interactions were obtained using the Transcriptional Regulatory Relationships Unraveled by Sentence-based Text mining (TRRUST) database Version 2 [17]. The TF-target DEG interaction networks, respectively, for the DEGs involved in the EC-induced NSPC alteration and NSPC-induced EC alteration, were constructed based on these TF-target DEG 


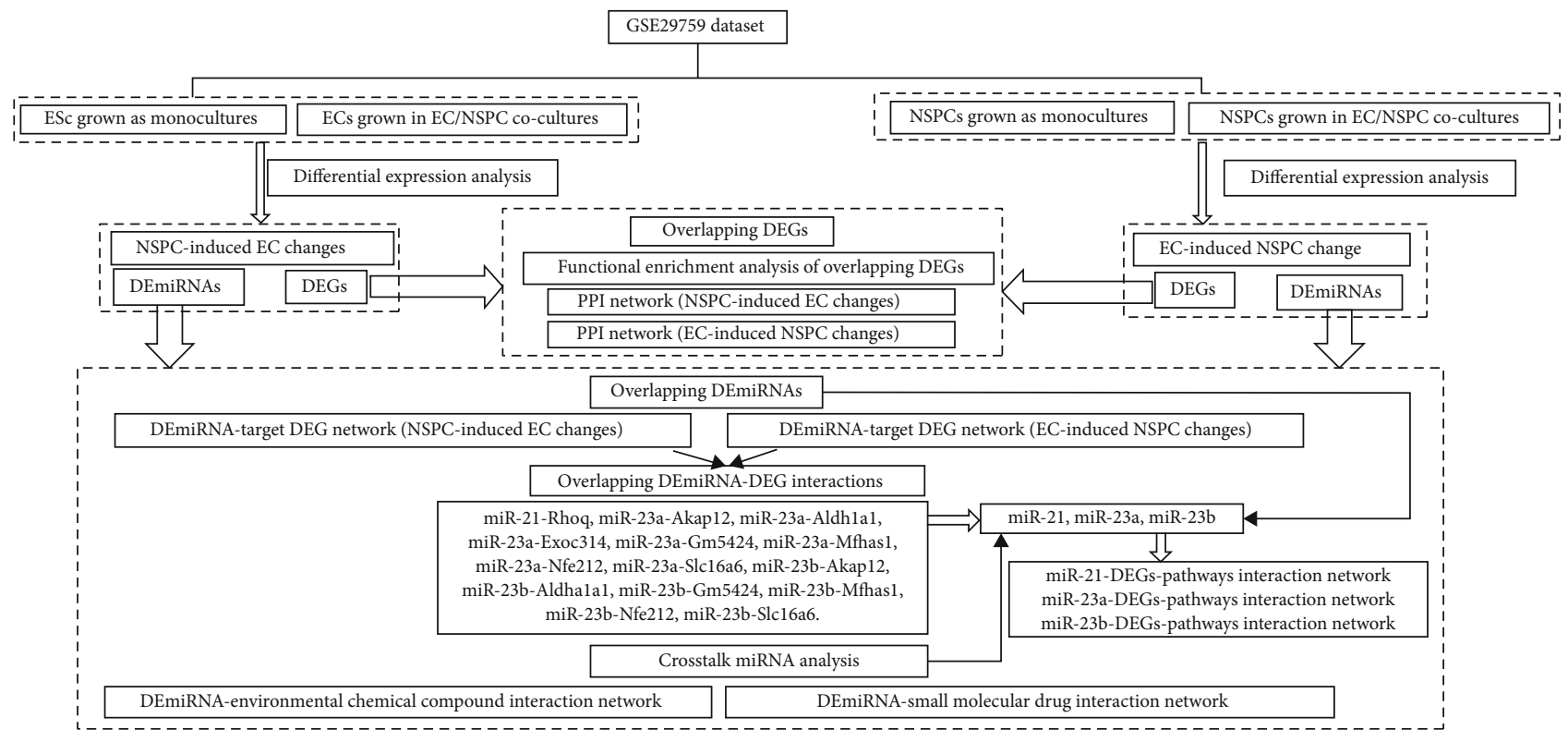

FIGURE 1: The flowchart of the present study.

TABLE 1: The number of up- and downregulated DEGs and DEmiRNAs that are, respectively, involved in NSPC-induced EC alterations and EC-induced NSPC alterations.

\begin{tabular}{|c|c|c|c|c|c|c|}
\hline \multirow[b]{2}{*}{$\begin{array}{l}\text { Coculture-induced genetic and epigenetic } \\
\text { alteration in one type of cells }\end{array}$} & \multicolumn{3}{|c|}{ Number of DEmRNAs (DEGs) } & \multicolumn{3}{|c|}{ Number of DEmiRNAs } \\
\hline & $\begin{array}{l}\text { Upregulated } \\
\text { DEGs }\end{array}$ & $\begin{array}{l}\text { Downregulated } \\
\text { DEGs }\end{array}$ & Total & $\begin{array}{l}\text { Upregulated } \\
\text { DEmiRNAs }\end{array}$ & $\begin{array}{l}\text { Downregulated } \\
\text { DEmiRNAs }\end{array}$ & Total \\
\hline NSPC-induced alterations in ECs & 399 & 345 & 744 & 5 & 13 & 18 \\
\hline EC-induced alterations in NSPCs & 425 & 710 & 1135 & 5 & 3 & 8 \\
\hline
\end{tabular}

interaction pairs. The topological characteristics (e.g., degree and betweenness centrality) of the nodes in the networks were also calculated.

2.6. Prediction of miRNA Targets. The experimentally validated gene targets of DEmiRNAs were obtained by three databases, including miRTarBase [18], miRecords [19], and TarBase v7 [20]. The computationally predicted gene targets of DEmiRNAs were obtained from eight databases including TargetScan [21], starBase [22], miRNAMap [23], miRDB [24], miRWalk [25], RNAhybrid [26], and miRanda [24]. Combining the experimentally validated and computationally predicted gene targets, a set of gene targets of the DEmiRNAs was obtained. From these, differentially expressed gene targets were identified and termed as miRNA-DEtarget. The DEmiRNA-target network involved in the EC-induced NSPC alteration and NSPC-induced EC alteration was constructed, respectively, using the clusterProfiler package in the $\mathrm{R}$ program [14]. The topological characteristics of nodes in this network were also calculated.

The DEmiRNA-DEtargets for each category were subjected to functional enrichment analysis in order to identify the signaling pathways with significant involvement. Corre- sponding miR-DEG-pathway interaction networks for each miRNA were constructed to examine DEtarget genes and their relevant enriched functional pathways.

2.7. The Relationship between DEmiRNAs and Small Molecular Drugs. The DEmiRNAs determined using the mouse genome were assigned corresponding Homo sapiens gene identities and further converted to the names of probes based on the HG-U133A platform. The human probe identities were uploaded to the Cmap web tool [27], and small molecular signatures of the selected DEmiRNAs were calculated by the following three formulas:

$$
\begin{gathered}
a=\operatorname{Max}_{j=1}^{t}\left[\frac{j}{t}-\frac{V(j)}{N}\right], \\
b=\operatorname{Max}_{j=1}^{t}\left[\frac{V(j)}{N}-\frac{(j-1)}{t}\right], \\
\mathrm{KS}_{\text {up/down }}=\left\{\begin{array}{l}
a(a>b) \\
-b(b>a)
\end{array}\right\} .
\end{gathered}
$$

The variable $t$ represents the number of DEmiRNAs which could be either upregulated or downregulated; $j$ represents the $j$ th gene based on the rank of the differential 

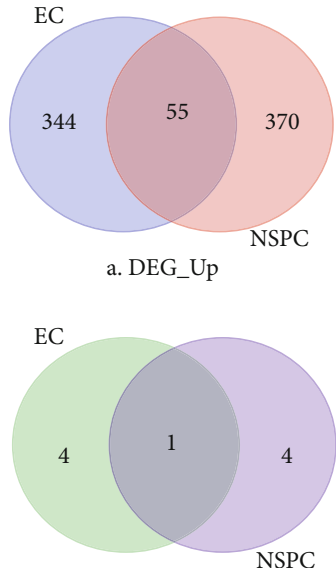

c. DEmiRNA_Up

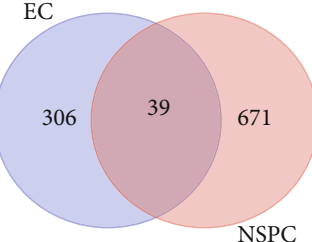

b. DEG_Down

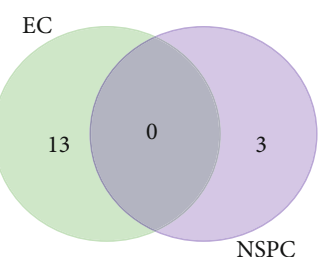

d. DEmiRNA_Down
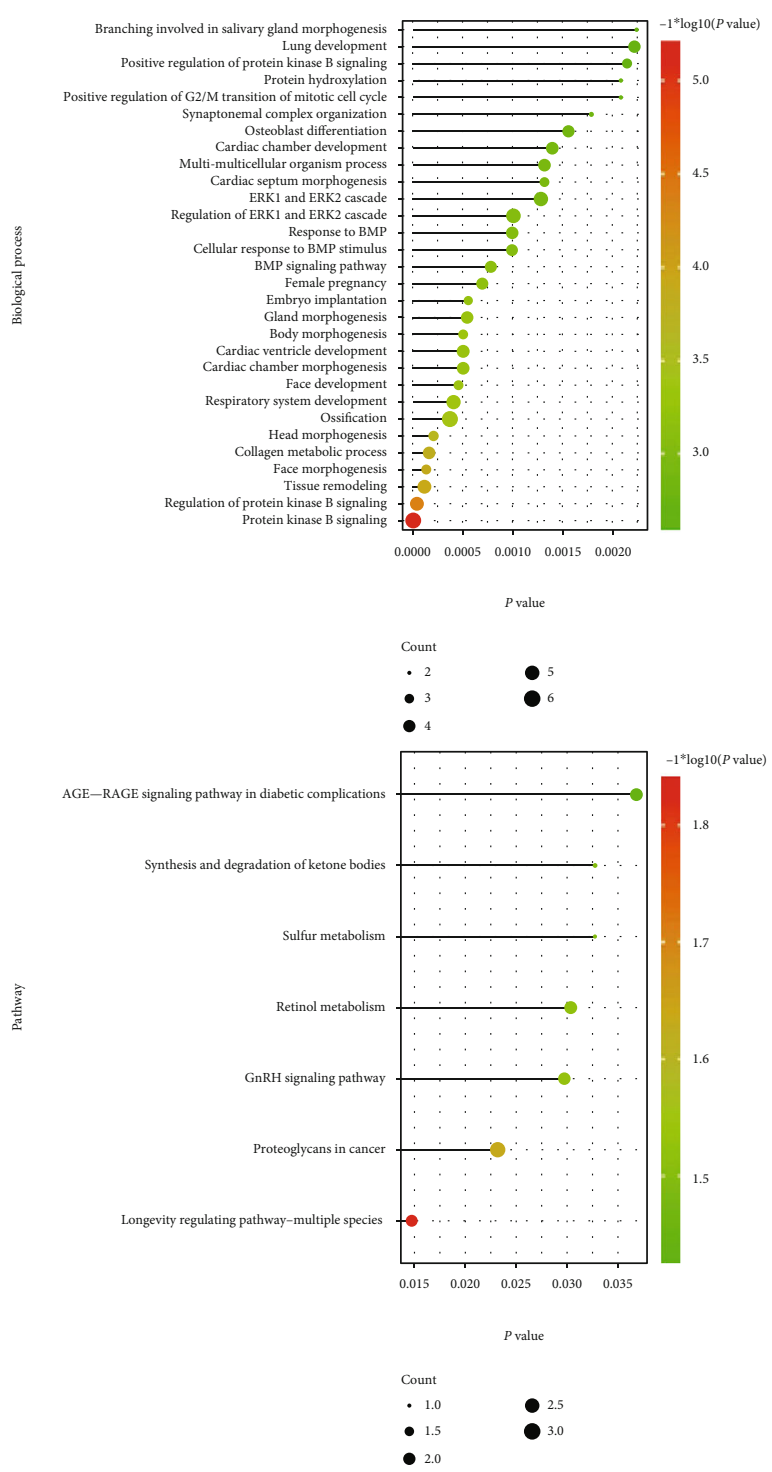

(b)

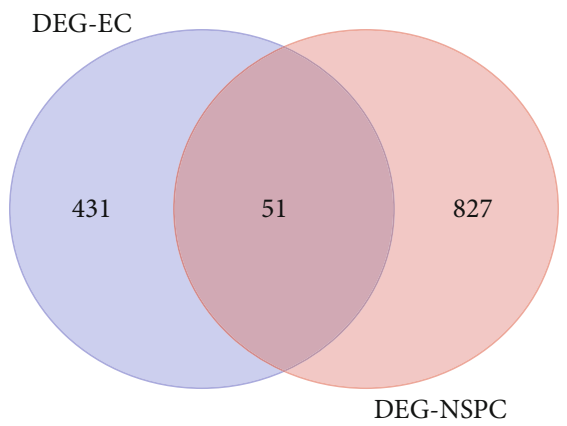

(c)

Figure 2: The DEGs identified to be involved in the EC-induced NSPC alterations and NSPC-induced EC alterations and the functions in which these DEGs were involved. (a) Venn diagram showing the overlapped DEGs and DEmiRNAs that hold the same expression patterns (either up- or downregulated) in EC-induced NSPC alterations and NSPC-induced EC alterations. (b) The top 30 BPs (A) and top signaling pathways (B) in which overlapped DEGs were enriched. The overlapped DEGs are shared between the NSPC-induced EC alterations and the EC-induced NSPC alterations. (c) The Venn diagram shows the DEGs overlapped between the DEGs involved in the EC-PPI network (PPI network involved in the NSPC-induced EC genetic alteration) and the NSPC-PPI network (PPI network involved in the EC-induced NSPC genetic alteration). 
TABLE 2: The top 30 DEGs involved in the NSPC-induced alterations in ECs, ranked by the ascending order of $P$ value.

\begin{tabular}{|c|c|c|c|c|c|c|c|}
\hline Gene & $\log \mathrm{FC}$ & AveExpr & $t$ value & $P$ value & Adj. $P$ value & $B$ value & Regulation pattern \\
\hline Matn2 & -0.95204 & 0.491256 & -10.0494 & $3.86 E-06$ & 0.025782 & 4.509474 & Down \\
\hline Fmod & -1.02587 & 0.511182 & -10.011 & $3.98 E-06$ & 0.025782 & 4.485352 & Down \\
\hline LOC102641248///Maf & -1.1636 & 0.572078 & -9.81371 & $4.69 E-06$ & 0.025782 & 4.359141 & Down \\
\hline Csf2 & 0.946405 & -0.49723 & 9.80391 & $4.73 E-06$ & 0.025782 & 4.352778 & $\mathrm{Up}$ \\
\hline Cyr61 & 1.916043 & -0.90785 & 9.163675 & $8.19 E-06$ & 0.035735 & 3.917772 & Up \\
\hline Rad54b & 0.884623 & -0.50864 & 8.718932 & $1.22 E-05$ & 0.038883 & 3.591553 & Up \\
\hline Hmgb3 & 1.027877 & -0.53931 & 8.697135 & $1.25 E-05$ & 0.038883 & 3.575027 & $\mathrm{Up}$ \\
\hline Srpx2 & -1.03053 & 0.491458 & -8.532 & $1.45 E-05$ & 0.039663 & 3.448146 & Down \\
\hline Ces1g & -1.12288 & 0.585271 & -8.39761 & $1.65 E-05$ & 0.040014 & 3.342658 & Down \\
\hline Glis3 & -0.82208 & 0.370708 & -8.27324 & $1.86 E-05$ & 0.040548 & 3.243204 & Down \\
\hline Negr1 & -0.85748 & 0.4562 & -8.03571 & $2.34 E-05$ & 0.041325 & 3.048246 & Down \\
\hline C1ra & -0.80236 & 0.398421 & -7.9815 & $2.47 E-05$ & 0.041325 & 3.002814 & Down \\
\hline Smarcal & -0.90427 & 0.471865 & -7.90938 & $2.65 E-05$ & 0.041325 & 2.941813 & Down \\
\hline Gmnn & 0.868301 & -0.43587 & 7.834033 & $2.86 E-05$ & 0.041325 & 2.877409 & Up \\
\hline Tex30 & 1.021454 & -0.5467 & 7.64017 & $3.48 E-05$ & 0.041325 & 2.708438 & Up \\
\hline Steap4 & -0.90097 & 0.356113 & -7.58391 & $3.68 E-05$ & 0.041325 & 2.658512 & Down \\
\hline Rbms3 & -0.79877 & 0.399764 & -7.5764 & $3.71 E-05$ & 0.041325 & 2.651814 & Down \\
\hline Cdo1 & -0.87123 & 0.459852 & -7.51396 & $3.96 E-05$ & 0.041325 & 2.595861 & Down \\
\hline Col3a1 & -0.72967 & 0.358484 & -7.49893 & $4.02 E-05$ & 0.041325 & 2.582317 & Down \\
\hline 3632451O06Rik & -0.99083 & 0.395715 & -7.37964 & $4.55 E-05$ & 0.041325 & 2.47378 & Down \\
\hline $\mathrm{C} 3$ & -0.8261 & 0.446644 & -7.36875 & $4.61 E-05$ & 0.041325 & 2.463774 & Down \\
\hline Thsd7a & -1.00741 & 0.566198 & -7.34217 & $4.74 E-05$ & 0.041325 & 2.439301 & Down \\
\hline Mad211 & 0.769878 & -0.43152 & 7.272629 & $5.10 E-05$ & 0.041325 & 2.374807 & $\mathrm{Up}$ \\
\hline Ccne2 & 0.891927 & -0.50969 & 7.268195 & $5.12 E-05$ & 0.041325 & 2.370674 & Up \\
\hline Adamts2 & -0.9331 & 0.460263 & -7.23757 & $5.29 E-05$ & 0.041325 & 2.342049 & Down \\
\hline AI506816 & 0.79492 & -0.41645 & 7.222375 & $5.38 E-05$ & 0.041325 & 2.327797 & $\mathrm{Up}$ \\
\hline Timm8a1 & 0.742468 & -0.41919 & 7.211119 & $5.44 E-05$ & 0.041325 & 2.317221 & Up \\
\hline Ccnb2 & 0.6736 & -0.32421 & 7.16572 & $5.71 E-05$ & 0.041325 & 2.274388 & Up \\
\hline Tyms///Tyms-ps & 0.676379 & -0.36727 & 7.152192 & $5.80 E-05$ & 0.041325 & 2.26157 & Up \\
\hline 5730414N17Rik & -0.79662 & 0.418908 & -7.14794 & $5.82 E-05$ & 0.041325 & 2.257534 & Down \\
\hline
\end{tabular}

expression; $N$ denotes the magnitude of the ranked small molecular signature; the element $V(j)$ of a vector $V$ is the position of the $j$ th target gene in the ordered small molecular signature. The corresponding $\mathrm{KS}_{\text {up }}$ and $\mathrm{KS}_{\text {down }}$ generated were integrated into the $S$ score as follows: $S$ is equal to 0 when $\mathrm{KS}_{\text {up }}$ and $\mathrm{KS}_{\text {down }}$ have the same sign and is equal to $\mathrm{KS}_{\text {up }}-\mathrm{KS}_{\text {down }}$ otherwise.

2.8. Predicting the Relationship between DEmiRNAs and Environmental Chemical Compounds. The relationship between environmental chemical compounds and DEmiRNAs was determined by downloading the corresponding interaction pairs from the Comparative Toxicogenomics Database (CTD) [28]. Highly correlated DEmiRNAsenvironmental chemical compounds were then determined by filtering using a hypergeometric test. The formula of the hypergeometric test is as follows:

$P(k, N, M, n)=\frac{\left(\begin{array}{c}M \\ k\end{array}\right) *\left(\begin{array}{c}N-M \\ n-k\end{array}\right)}{\left(\begin{array}{c}N \\ n\end{array}\right)}, \quad k=0,1,2,3, \cdots, M$.

$N$ represents the total number of DEmiRNAs; $n$ represents the number of DEmiRNAs corresponding to a specific chemical compound; $M$ represents the number of the target genes of a specific miRNA; $k$ represents the genes which 
TABLE 3: The top 30 DEGs involved in the EC-involved alterations in NSPCs, ranked by the ascending order of $P$ value.

\begin{tabular}{|c|c|c|c|c|c|c|c|}
\hline Gene & $\log \mathrm{FC}$ & AveExpr & $t$ value & $P$ value & Adj. $P$ value & $B$ value & Regulation pattern \\
\hline Pcolce2 & -3.29485 & 1.6351 & -45.4871 & $2.08 E-11$ & $4.55 E-07$ & 13.2377 & Down \\
\hline Dbp & -2.00859 & 1.062409 & -26.2015 & $2.14 E-09$ & $2.34 E-05$ & 11.11723 & Down \\
\hline AU020206 & -1.63296 & 0.821066 & -21.5827 & $1.08 E-08$ & $7.32 E-05$ & 10.05187 & Down \\
\hline F3 & -1.6611 & 0.850706 & -20.9105 & $1.41 E-08$ & $7.32 E-05$ & 9.863187 & Down \\
\hline Tpil & -1.68121 & 0.830618 & -20.4734 & $1.68 E-08$ & $7.32 E-05$ & 9.73502 & Down \\
\hline $\mathrm{Gm} 2115$ & -1.53464 & 0.719453 & -19.4163 & $2.60 E-08$ & $9.46 E-05$ & 9.405935 & Down \\
\hline Ldha & -1.78095 & 0.900045 & -18.2662 & $4.32 E-08$ & 0.000134 & 9.014231 & Down \\
\hline Sorl1 & -1.45704 & 0.706881 & -17.7044 & $5.59 E-08$ & 0.000135 & 8.808873 & Down \\
\hline Dct & -1.30765 & 0.669351 & -17.6998 & $5.60 E-08$ & 0.000135 & 8.807132 & Down \\
\hline Klf9 & -1.86306 & 0.937896 & -17.4915 & $6.17 E-08$ & 0.000135 & 8.728473 & Down \\
\hline Pgk1 & -1.18385 & 0.609345 & -16.5411 & $9.78 E-08$ & 0.000194 & 8.351213 & Down \\
\hline Gypc & -1.22407 & 0.595138 & -16.039 & $1.26 E-07$ & 0.000207 & 8.139031 & Down \\
\hline Aldoa & -1.26041 & 0.65786 & -15.9803 & $1.30 E-07$ & 0.000207 & 8.113603 & Down \\
\hline Plekhf1 & -1.317 & 0.690027 & -15.8197 & $1.41 E-07$ & 0.000207 & 8.043409 & Down \\
\hline 4-Sep & -1.14394 & 0.579925 & -15.6971 & $1.50 E-07$ & 0.000207 & 7.989137 & Down \\
\hline Aldh1a1 & -2.80632 & 1.506176 & -15.6785 & $1.52 E-07$ & 0.000207 & 7.980857 & Down \\
\hline Plin 3 & -1.2668 & 0.589403 & -15.1096 & $2.06 E-07$ & 0.000264 & 7.720663 & Down \\
\hline Parp9 & -1.23876 & 0.648245 & -14.7499 & $2.50 E-07$ & 0.000303 & 7.549197 & Down \\
\hline Cldn12 & -1.28813 & 0.622674 & -14.0937 & $3.63 E-07$ & 0.000408 & 7.221524 & Down \\
\hline Gpil & -1.00716 & 0.517518 & -14.0417 & $3.74 E-07$ & 0.000408 & 7.194653 & Down \\
\hline Htra1 & -1.19764 & 0.56397 & -13.8878 & $4.09 E-07$ & 0.000409 & 7.114509 & Down \\
\hline Srd5a1 & -1.16578 & 0.561656 & -13.8718 & $4.13 E-07$ & 0.000409 & 7.106129 & Down \\
\hline Dlx5 & 1.127851 & -0.58715 & 13.43145 & $5.37 E-07$ & 0.000478 & 6.87001 & $\mathrm{Up}$ \\
\hline ND5 & 1.058484 & -0.52334 & 13.36773 & $5.58 E-07$ & 0.000478 & 6.835023 & Up \\
\hline Rbp1 & -1.12775 & 0.585747 & -13.3416 & $5.67 E-07$ & 0.000478 & 6.820608 & Down \\
\hline Atp1b2 & -1.03892 & 0.500454 & -13.3344 & $5.69 E-07$ & 0.000478 & 6.816639 & Down \\
\hline Clybl & -1.27083 & 0.618039 & -13.1367 & $6.42 E-07$ & 0.000519 & 6.706417 & Down \\
\hline Sox8 & -1.02853 & 0.485142 & -12.9808 & $7.08 E-07$ & 0.000551 & 6.617969 & Down \\
\hline Srprb///Trf & -1.18478 & 0.624131 & -12.8262 & $7.80 E-07$ & 0.000586 & 6.52904 & Down \\
\hline Hspb6 & -0.93188 & 0.490732 & -12.7078 & $8.40 E-07$ & 0.000594 & 6.459985 & Down \\
\hline
\end{tabular}

not only belong to the target genes of DEmiRNAs but also are related to chemical compounds.

2.9. Crosstalk miRNA Analysis. The crosstalk miRNAs were explored using the "Meet/Min" score based on the gene targets, small molecules (DEmiRNA-molecule interaction pairs), environmental chemical compounds, signaling pathways, and biological processes in GO terms. The "Meet/Min" score was calculated as follows:

$$
\operatorname{Score}(i, j)=\frac{|\operatorname{targets}(i) \cap \operatorname{targets}(j)|}{\min (|\operatorname{targets}(i)|, \operatorname{targets}(j) \mid)}
$$

For one miRNA pair, $i$ and $j$, their differentially expressed target gene sets were $\operatorname{targets}(i)$ and $\operatorname{targets}(j)$, respectively.
The number of common differentially expressed targets of the two miRNAs was divided by the size of the smaller target set. Interaction pairs of miRNAs were identified through filtering based on interaction with a measurement of 0 or without common features (e.g., environmental chemical compounds, small molecular drugs). Thereafter, a miRNA crosstalk network was constructed.

\section{Results}

3.1. The Flowchart of the Present Study. The study design of the present research is shown in Figure 1. As shown in Figure 1, the differential expression analysis was performed to investigate the DEGs and DEmiRNAs involved in the NSPC-induced EC alteration and EC-induced NSPC 


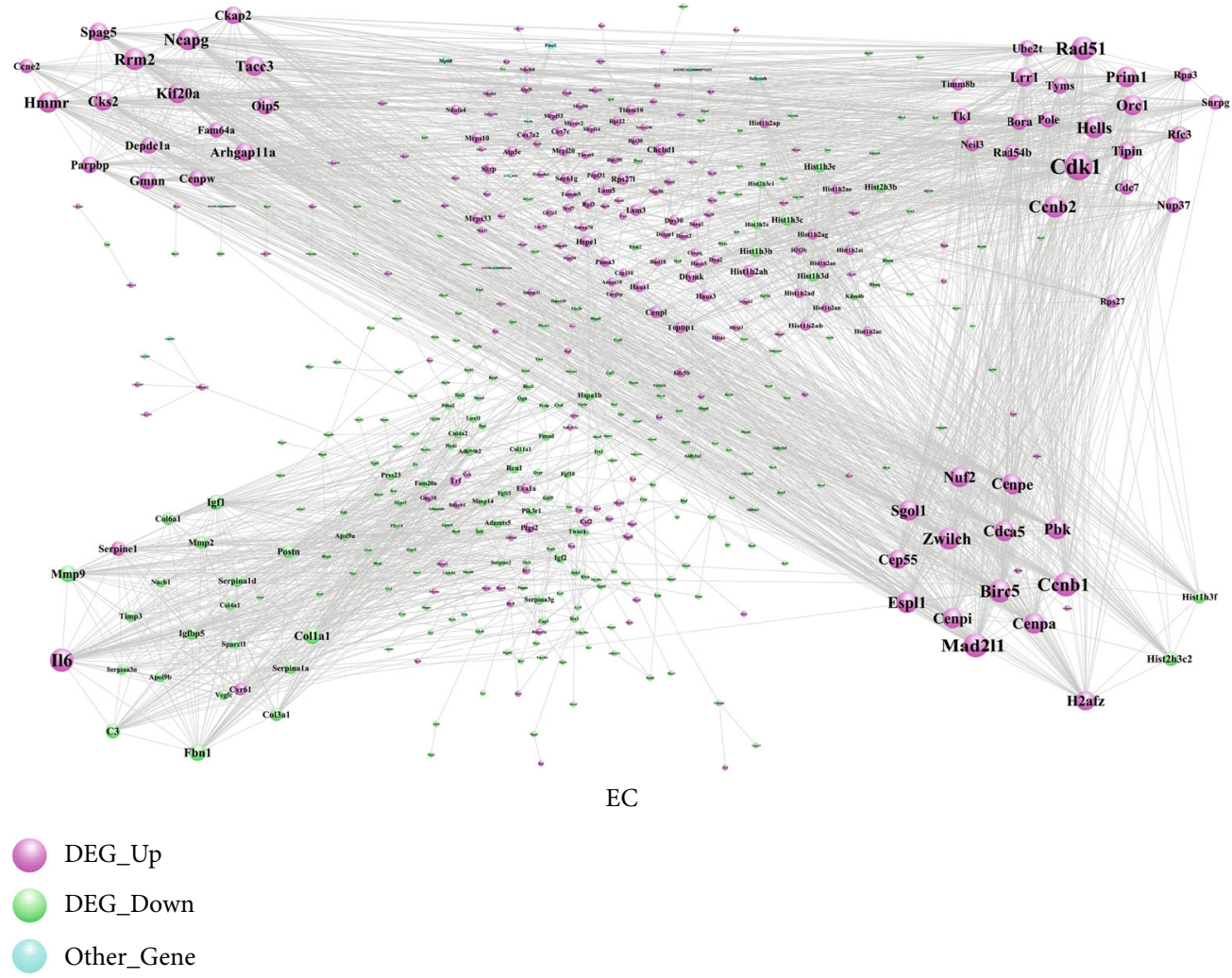

(a)

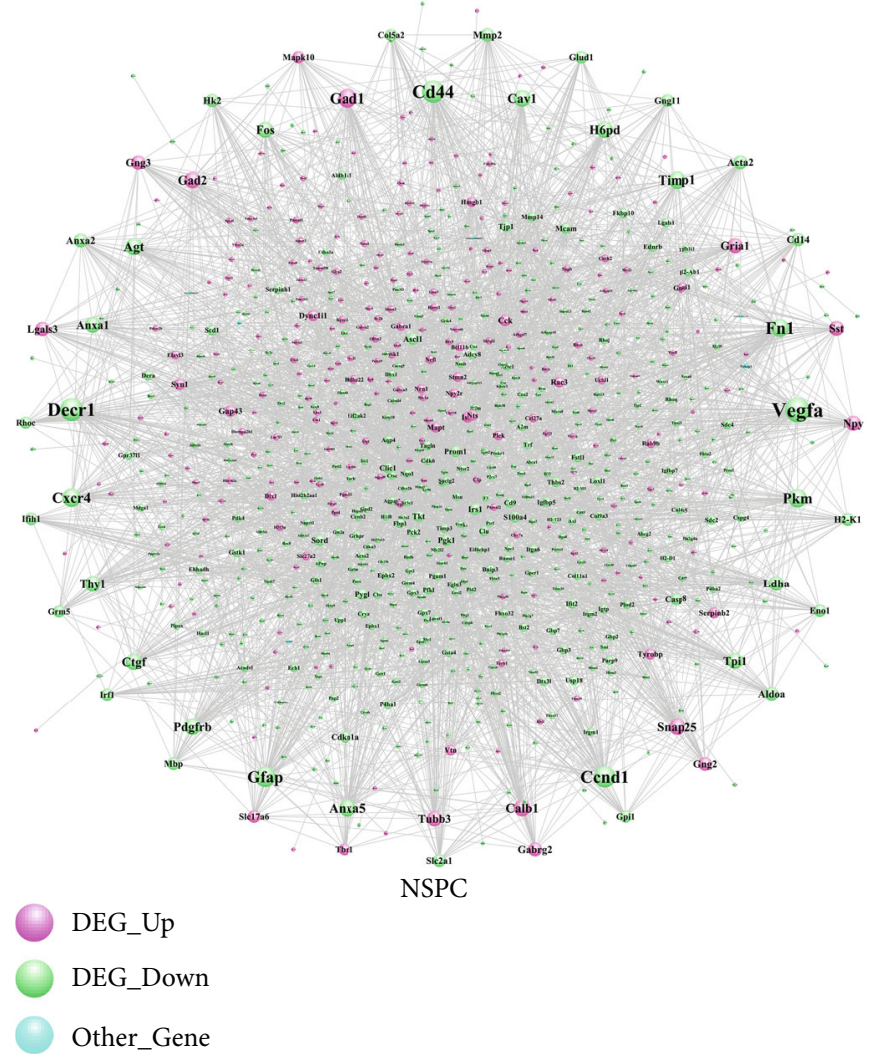

(b)

FIGURE 3: The PPI network of DEGs involved in NSPC-induced EC alteration (a) and DEGs involved in EC-induced NSPC alteration (b). Other genes are non-DEGs. 
TABLE 4: The topological characteristics of the top 30 nodes in the PPI network of NSPC-induced EC genetic alterations. NA means not overlapped between the EC-PPI and NSPC-PPI networks.

\begin{tabular}{|c|c|c|c|c|c|c|c|}
\hline DEGs & Overlap & Regulate & Degree & $\begin{array}{c}\text { Average shortest path } \\
\text { length }\end{array}$ & $\begin{array}{c}\text { Betweenness } \\
\text { centrality }\end{array}$ & $\begin{array}{l}\text { Closeness } \\
\text { centrality }\end{array}$ & $\begin{array}{c}\text { Topological } \\
\text { coefficient }\end{array}$ \\
\hline Cdk1 & NA & $\mathrm{Up}$ & 72 & 2.737069 & 0.056051 & 0.365354 & 0.179894 \\
\hline Mad2l1 & NA & Up & 63 & 2.844828 & 0.019572 & 0.351515 & 0.211029 \\
\hline Ccnb1 & NA & Up & 60 & 2.706897 & 0.084386 & 0.369427 & 0.195539 \\
\hline Il6 & NA & Up & 58 & 2.872845 & 0.111663 & 0.348087 & 0.08938 \\
\hline Rad51 & NA & Up & 58 & 2.855603 & 0.04335 & 0.350189 & 0.232014 \\
\hline Birc5 & NA & Up & 56 & 2.732759 & 0.058321 & 0.365931 & 0.213803 \\
\hline Ccnb2 & NA & Up & 54 & 2.922414 & 0.006045 & 0.342183 & 0.255649 \\
\hline Ncapg & NA & Up & 52 & 3.032328 & 0.008236 & 0.32978 & 0.282051 \\
\hline Rrm2 & NA & Up & 52 & 2.931034 & 0.018658 & 0.341176 & 0.262327 \\
\hline Zwilch & NA & Up & 52 & 2.974138 & 0.008695 & 0.336232 & 0.26455 \\
\hline Espl1 & NA & Up & 51 & 2.961207 & 0.010195 & 0.3377 & 0.265609 \\
\hline Hells & NA & Up & 51 & 2.967672 & 0.006265 & 0.336964 & 0.268318 \\
\hline Cdca5 & NA & $\mathrm{Up}$ & 49 & 2.971983 & 0.002445 & 0.336476 & 0.279941 \\
\hline Cenpa & NA & Up & 49 & 2.997845 & 0.016442 & 0.333573 & 0.260875 \\
\hline Cenpe & NA & Up & 49 & 2.961207 & 0.018044 & 0.3377 & 0.257355 \\
\hline $\mathrm{Hmmr}$ & NA & Up & 49 & 3.040948 & 0.009178 & 0.328845 & 0.288843 \\
\hline $\mathrm{Pbk}$ & NA & Up & 49 & 3.006466 & 0.002026 & 0.332616 & 0.287645 \\
\hline Prim1 & NA & Up & 49 & 2.935345 & 0.019553 & 0.340675 & 0.251913 \\
\hline Nuf2 & NA & Up & 48 & 2.974138 & 0.00309 & 0.336232 & 0.275905 \\
\hline Sgol1 & NA & Up & 48 & 2.997845 & 0.002882 & 0.333573 & 0.28125 \\
\hline Cenpi & NA & Up & 47 & 2.99569 & 0.017411 & 0.333813 & 0.26534 \\
\hline Kif20a & NA & Up & 47 & 3.0625 & 0.001547 & 0.326531 & 0.30589 \\
\hline Orc1 & NA & $\mathrm{Up}$ & 47 & 2.989224 & 0.022176 & 0.334535 & 0.25024 \\
\hline Tacc3 & NA & $\mathrm{Up}$ & 46 & 2.939655 & 0.025243 & 0.340176 & 0.287225 \\
\hline $\mathrm{H} 2 \mathrm{afz}$ & NA & $\mathrm{Up}$ & 45 & 3.002155 & 0.040379 & 0.333094 & 0.188816 \\
\hline Cep55 & NA & $\mathrm{Up}$ & 44 & 3.071121 & 0.000625 & 0.325614 & 0.323656 \\
\hline Cks2 & NA & $\mathrm{Up}$ & 43 & 2.980603 & 0.001636 & 0.335503 & 0.301077 \\
\hline Lrr1 & NA & $\mathrm{Up}$ & 43 & 3.047414 & 0.025254 & 0.328147 & 0.28961 \\
\hline Spag5 & NA & $\mathrm{Up}$ & 42 & 3.096983 & 0.000598 & 0.322895 & 0.327866 \\
\hline Arhgap11a & NA & Up & 41 & 3.090517 & 0.043119 & 0.32357 & 0.316706 \\
\hline
\end{tabular}

alteration. As regards DEGs, the DEGs overlapped between DEGs involved in NSPC-induced EC alteration and ECinduced NSPC alteration were identified, and then the functional enrichment analysis was performed to investigate the biological processes and signaling pathways in which these overlapping DEGs were involved. Based on the DEGs, respectively, involved in the NSPC-induced EC alteration and EC-induced NSPC alteration, the corresponding PPI network was constructed. As regards DEmiRNAs, the DEmiRNAs overlapped between DEmiRNAs expressed in the NSPC-induced EC alteration and EC-induced NSPC alteration were identified to be mmu-miR-23a, mmu-miR23b, and mmu-miR-210. Afterward, the DEmiRNA-DEG interaction networks were constructed, respectively, for the NSPC-induced EC alteration and EC-induced NSPC alteration, from which the overlapping DEmiRNA-DEG interaction pairs were found. The crosstalk miRNA analysis was performed and validated the crosstalk role of three DEmiRNAs (mmu-miR-23a, mmu-miR-23b, and mmu-miR-210) in the interaction between ECs and NSPCs. The corresponding miRNA-DEG-pathway network, respectively, for these three DEmiRNAs was constructed. In addition, the chemical compounds and small molecular drugs which regulate the expression patterns of DEmiRNAs were also identified by constructing the DEmiRNA-environmental chemical compound interaction network and DEmiRNA-small molecular drug interaction network.

3.2. Identification of DEGs and DEmiRNAs. Table 1 and Figure 2(a) show that 94 DEGs consisting of 55 upregulated DEGs and 39 downregulated DEGs were overlapping. Tables 2 and 3 list the differential expression values (e.g., $\log$ FC, AveExpr, $t$ value, $P$ value, adj. $P$ value, $B$ value, and regulation pattern (up/down)) of the top 30 DEGs which 
TABLE 5: The topological characteristics of the top 30 nodes in the PPI network of EC-induced NSPC genetic alterations. NA means not overlapped between the EC-PPI and NSPC-PPI networks.

\begin{tabular}{|c|c|c|c|c|c|c|c|}
\hline Gene & Overlap & Regulate & Degree & Average shortest path length & Betweenness centrality & Closeness centrality & Topological coefficient \\
\hline Gapdh & NA & Down & 129 & 2.303797 & 0.21952 & 0.434066 & 0.040603 \\
\hline Fn1 & NA & Down & 83 & 2.586881 & 0.062983 & 0.386566 & 0.054676 \\
\hline Vegfa & NA & Down & 64 & 2.638665 & 0.041079 & 0.37898 & 0.063087 \\
\hline Decr1 & NA & Down & 59 & 2.609896 & 0.066189 & 0.383157 & 0.059219 \\
\hline $\mathrm{Cd} 44$ & NA & Down & 57 & 2.693901 & 0.029026 & 0.371209 & 0.069522 \\
\hline Ccnd1 & NA & Down & 54 & 2.703107 & 0.040282 & 0.369945 & 0.065015 \\
\hline Gfap & NA & Down & 51 & 2.663982 & 0.042334 & 0.375378 & 0.070175 \\
\hline Gad1 & NA & Up & 47 & 2.837745 & 0.023163 & 0.352393 & 0.075753 \\
\hline Cxcr4 & NA & Down & 45 & 2.805524 & 0.015376 & 0.35644 & 0.091761 \\
\hline $\mathrm{Pkm}$ & NA & Down & 45 & 2.780207 & 0.042856 & 0.359685 & 0.072881 \\
\hline Anxa5 & NA & Down & 41 & 2.817031 & 0.007322 & 0.354984 & 0.091351 \\
\hline Timp1 & NA & Down & 41 & 2.805524 & 0.010114 & 0.35644 & 0.09083 \\
\hline Agt & NA & Down & 40 & 2.857307 & 0.014755 & 0.34998 & 0.0945 \\
\hline Cav1 & NA & Down & 40 & 2.788262 & 0.021742 & 0.358646 & 0.081866 \\
\hline H6pd & Overlap & Down & 39 & 2.905639 & 0.018002 & 0.344158 & 0.081197 \\
\hline Calb1 & NA & Up & 39 & 2.827388 & 0.022978 & 0.353683 & 0.082931 \\
\hline Ctgf & NA & Down & 38 & 2.79977 & 0.019484 & 0.357172 & 0.08851 \\
\hline Pdgfrb & NA & Down & 38 & 2.840046 & 0.010704 & 0.352107 & 0.092062 \\
\hline Snap25 & NA & Up & 38 & 2.840046 & 0.026457 & 0.352107 & 0.071554 \\
\hline Fos & NA & Down & 37 & 2.757192 & 0.026678 & 0.362688 & 0.086654 \\
\hline Gad2 & NA & Up & 37 & 2.842348 & 0.016537 & 0.351822 & 0.0868 \\
\hline Anxa1 & NA & Down & 36 & 2.912543 & 0.010209 & 0.343343 & 0.091158 \\
\hline Tpil & NA & Down & 36 & 2.905639 & 0.015808 & 0.344158 & 0.086608 \\
\hline Grial & NA & Up & 35 & 2.837745 & 0.033782 & 0.352393 & 0.079529 \\
\hline Ldha & NA & Down & 35 & 2.933257 & 0.010636 & 0.340918 & 0.096276 \\
\hline Thy1 & NA & Down & 35 & 2.886076 & 0.018426 & 0.346491 & 0.090796 \\
\hline Tubb3 & NA & Up & 35 & 2.829689 & 0.013785 & 0.353396 & 0.087982 \\
\hline Npy & NA & Up & 34 & 2.906789 & 0.010344 & 0.344022 & 0.099831 \\
\hline Sst & NA & Up & 34 & 2.921749 & 0.012787 & 0.342261 & 0.101852 \\
\hline Gng3 & NA & Up & 32 & 3.179517 & 0.007673 & 0.314513 & 0.107118 \\
\hline
\end{tabular}

were, respectively, differentially expressed in the NSPCinduced EC alteration and EC-induced NSPC alteration. Three DEmiRNAs found to be overlapped between the ECinduced NSPC alteration and NSPC-induced EC alteration categories (i.e., mmu-miR-23a, mmu-miR-23b, and mmumiR-210) were selected for further investigation. The expression pattern of mmu-miR-210 was similar in both ECinduced NSPC alterations and NSPC-induced EC alterations. However, the expression patterns of mmu-miR-23a and mmu-miR-23b differed in both situations.

3.3. Functions of Overlapping DEGs. Figure 2(b) depicts the biological processes and pathways in which overlapping DEGs were enriched. As seen in Figure 2(b) A, the overlapping DEGs were found to be involved in several biological processes, for instance, positive regulation of protein kinase $\mathrm{B}$ (PKB, also known as Akt), ERK1/2 cascade, response to
BMP, collagen metabolic process, and tissue remodeling. The overlapping DEGs were found to be involved in several signaling pathways including synthesis and degradation of ketone bodies, sulfur metabolism, retinol metabolism, and GnRH signaling (Figure 2(b) B).

3.4. EC-PPI Network and NSPC-PPI Network. The PPI network consisting of DEGs involved in NSPC-induced EC alteration (EC-PPI network) was constructed and included 482 nodes with 2,679 edges (Figure 3(a)), while the PPI network consisting of DEGs involved in EC-induced NSPC alteration (NSPC-PPI network) showed 878 nodes and 3,795 edges (Figure 3(b)). The characteristics of the top 30 nodes of EC-PPI as well as NSPC-PPI network are listed in descending order of degree (Tables 4 and 5).

By construction of a Venn diagram, 51 DEGs were overlapping between the EC-PPI network and the NSPC-PPI 
TABLE 6: The topological characteristics of overlapped DEGs in the PPI network of NSPC-induced EC genetic alterations. The overlapped DEGs mean these DEGs were overlapped between the PPI network of NSPC-induced EC alteration and the PPI network of EC-induced NSPC alteration.

\begin{tabular}{|c|c|c|c|c|c|c|}
\hline Overlapped DEGs & Regulate & Degree & Average shortest path length & Betweenness centrality & Closeness centrality & Topological coefficient \\
\hline Timp3 & Down & 21 & 3.394397 & 0.012355 & 0.294603 & 0.193591 \\
\hline Mmp14 & Down & 15 & 3.321121 & 0.006668 & 0.301103 & 0.213333 \\
\hline Col11a1 & Down & 14 & 3.681034 & 0.000966 & 0.271663 & 0.265873 \\
\hline $\mathrm{H} 3 \mathrm{f} 3 \mathrm{~b}$ & $\mathrm{Up}$ & 13 & 3.793103 & $3.40 E-05$ & 0.263636 & 0.329522 \\
\hline Loxl1 & Down & 13 & 3.568966 & 0.001462 & 0.280193 & 0.254605 \\
\hline Ccp110 & Up & 12 & 3.497845 & 0.007609 & 0.28589 & 0.324906 \\
\hline Rpl30 & $\mathrm{Up}$ & 12 & 3.715517 & 0.001937 & 0.269142 & 0.208333 \\
\hline Irs1 & Down & 11 & 3.103448 & 0.01987 & 0.322222 & 0.171937 \\
\hline Aldh1a1 & Down & 9 & 3.403017 & 0.022894 & 0.293857 & 0.139918 \\
\hline P4ha2 & Down & 9 & 3.913793 & 0.00226 & 0.255507 & 0.381313 \\
\hline Cck & Up & 8 & 3.579741 & 0.011327 & 0.27935 & 0.194444 \\
\hline Plod2 & Down & 8 & 4.118534 & $7.55 E-06$ & 0.242805 & 0.428977 \\
\hline Rhoq & Down & 8 & 4.008621 & 0.012817 & 0.249462 & 0.156863 \\
\hline H6pd & Down & 7 & 3.534483 & 0.017785 & 0.282927 & 0.159664 \\
\hline Luc7l3 & Up & 7 & 3.571121 & 0.009536 & 0.280024 & 0.188065 \\
\hline Dlx5 & $\mathrm{Up}$ & 6 & 3.784483 & 0.007836 & 0.264237 & 0.215278 \\
\hline Rcn3 & Down & 6 & 3.525862 & 0.008417 & 0.283619 & 0.330247 \\
\hline F3 & Down & 5 & 3.56681 & $7.31 E-06$ & 0.280363 & 0.413514 \\
\hline Hspa2 & Down & 5 & 3.221983 & 0.000827 & 0.310368 & 0.275229 \\
\hline $\operatorname{Tgm} 2$ & Down & 5 & 3.538793 & 0.005005 & 0.282582 & 0.379221 \\
\hline Aldh1a7 & Down & 4 & 4.165948 & 0.000317 & 0.240041 & 0.416667 \\
\hline $\operatorname{Irgm} 2$ & Down & 4 & 4.290948 & 0.000557 & 0.233049 & 0.3125 \\
\hline Smad6 & Down & 4 & 3.928879 & 0.002692 & 0.254526 & 0.289474 \\
\hline Bmper & Down & 3 & 4.810345 & 0.000753 & 0.207885 & 0.433333 \\
\hline Daam2 & Down & 3 & 3.87931 & 0.008654 & 0.257778 & 0.333333 \\
\hline Rras & Down & 3 & 4.3125 & 0.000343 & 0.231884 & 0.439394 \\
\hline Syde1 & Down & 3 & 4.939655 & 0.000344 & 0.202443 & 0.545455 \\
\hline Tns1 & Down & 3 & 4.286638 & 0.002258 & 0.233283 & 0.355556 \\
\hline Fcgrt & Down & 2 & 4.157328 & 0 & 0.240539 & 0.576923 \\
\hline Rin2 & Down & 2 & 4.681034 & 0.000218 & 0.213628 & 0.5 \\
\hline Sdpr & Down & 2 & 4.275862 & 0.000187 & 0.233871 & 0.5 \\
\hline Slc2a10 & Down & 2 & 4.176724 & 0 & 0.239422 & 0.738095 \\
\hline
\end{tabular}

network (Figure 2(c)). The topological features of the overlapping DEGs in the EC-PPI network and the NSPC-PPI network are listed in Tables 6 and 7, respectively.

3.5. The Transcription Factor-Target DEG Interaction Network. The TF-target DEG network was constructed, respectively, for the DEGs involved in the NSPC-induced EC alteration (Figure 4(a)) and EC-induced NPSC alteration (Figure 4(b)). Figure 4(a) consists of 285 nodes and 363 edges, while Figure 4(b) consists of 451 nodes and 668 edges. As shown in Figure 4(a) and Table 8, several TFs (e.g., Sp1, Nfkb1, Trp53, Jun, Twist1, and Stat3) were identified to be hub transcription factors targeting the greatest number of DEGs involved in the NSPC-induced EC alteration. As shown in Figure 4(b) and Table 9, several TFs (e.g., Ccnd1, Sp1, Fos, Hes1, Nfkb1, and Cebpb) were identified to be hub transcription factors targeting the greatest number of DEGs involved in the EC-induced NPSC alteration.

3.6. The DEmiRNAs-Target DEGs and Their Functions. The number of experimentally validated, computationally predicted, and total miRNA-DEtargets for ECs and NSPCs is listed in Table 10. As evident here, 14 interaction pairs of miRNA-DEtargets were determined. The DEmiRNA-target network involved in NSPC-induced EC alteration was constructed and included 5,916 nodes with 22,664 edges (Figure 5(a)), while the DEmiRNA-target network involved in NSPC-induced EC alteration showed 4,962 nodes and 
TABLE 7: The topological characteristics of overlapped DEGs in the PPI network of EC-induced NSPC genetic alterations. The overlapped DEGs mean these DEGs were overlapped between the PPI network of NSPC-induced EC alteration and the PPI network of EC-induced NSPC alteration.

\begin{tabular}{|c|c|c|c|c|c|c|}
\hline Overlapped DEGs & Regulate & Degree & Average shortest path length & Betweenness centrality & Closeness centrality & Topological coefficient \\
\hline H6pd & Down & 39 & 2.905639 & 0.018002 & 0.344158 & 0.081197 \\
\hline Mmp2 & Down & 30 & 2.905639 & 0.009375 & 0.344158 & 0.111791 \\
\hline Cck & Up & 24 & 3.161105 & 0.002392 & 0.316345 & 0.14388 \\
\hline Irs1 & Down & 24 & 2.90794 & 0.011375 & 0.343886 & 0.090661 \\
\hline Igfbp5 & Down & 18 & 3.002302 & 0.001391 & 0.333078 & 0.145524 \\
\hline Loxl1 & Down & 18 & 3.331415 & 0.002588 & 0.300173 & 0.154147 \\
\hline Mmp14 & Down & 18 & 2.958573 & 0.006212 & 0.338001 & 0.147655 \\
\hline Aldhla1 & Down & 16 & 3.079402 & 0.008487 & 0.324738 & 0.124419 \\
\hline Plod2 & Down & 16 & 3.535098 & 0.002197 & 0.282878 & 0.168269 \\
\hline Col11a1 & Down & 15 & 3.464902 & 0.007219 & 0.288608 & 0.154386 \\
\hline Timp3 & Down & 15 & 3.065593 & 0.004764 & 0.326201 & 0.152648 \\
\hline $\operatorname{Irgm} 2$ & Down & 14 & 3.910242 & $4.82 E-05$ & 0.255739 & 0.348397 \\
\hline P4ha2 & Down & 14 & 3.637514 & 0.001633 & 0.274913 & 0.177721 \\
\hline Rhoq & Down & 13 & 3.673188 & 0.001094 & 0.272243 & 0.173382 \\
\hline F3 & Down & 11 & 3.150748 & 0.00066 & 0.317385 & 0.188776 \\
\hline Dlx5 & $\mathrm{Up}$ & 10 & 3.500575 & 0.001701 & 0.285667 & 0.221359 \\
\hline Hspa2 & Down & 10 & 3.356732 & 0.009675 & 0.297909 & 0.113084 \\
\hline Luc7l3 & $\mathrm{Up}$ & 9 & 3.771001 & 0.004972 & 0.265182 & 0.17284 \\
\hline Syde1 & Down & 9 & 3.562716 & 0.005367 & 0.280685 & 0.202822 \\
\hline Tgm2 & Down & 9 & 3.103567 & 0.002864 & 0.32221 & 0.194714 \\
\hline Rras & Down & 8 & 3.651323 & 0.00461 & 0.273873 & 0.227113 \\
\hline Sdpr & Down & 8 & 3.372842 & 0.002511 & 0.296486 & 0.180118 \\
\hline Aldh1a7 & Down & 7 & 3.686997 & 0.000587 & 0.271223 & 0.221254 \\
\hline Ccp110 & $\mathrm{Up}$ & 7 & 3.982739 & 0.002565 & 0.251084 & 0.159664 \\
\hline Cdc7 & $\mathrm{Up}$ & 7 & 3.821634 & 0.000152 & 0.261668 & 0.313589 \\
\hline Rcn3 & Down & 7 & 3.697353 & 0.000432 & 0.270464 & 0.249433 \\
\hline Tns1 & Down & 7 & 3.397008 & 0.005802 & 0.294377 & 0.188729 \\
\hline Zcchc12 & $\mathrm{Up}$ & 6 & 3.775604 & 0.006142 & 0.264858 & 0.217391 \\
\hline Akap12 & Down & 5 & 3.422325 & 0.002599 & 0.292199 & 0.261111 \\
\hline Daam2 & Down & 5 & 3.658228 & 0.000694 & 0.273356 & 0.303226 \\
\hline $\mathrm{H} 3 \mathrm{f} 3 \mathrm{~b}$ & $\mathrm{Up}$ & 5 & 4.362486 & $6.29 E-05$ & 0.229227 & 0.5125 \\
\hline Rpl30 & Up & 5 & 4.084005 & 0.000687 & 0.244858 & 0.3 \\
\hline Sema3c & Down & 5 & 3.528193 & 0.002593 & 0.283431 & 0.2075 \\
\hline Smad6 & Down & 5 & 3.510932 & 0.001004 & 0.284825 & 0.284848 \\
\hline Bmper & Down & 4 & 4.210587 & 0.00075 & 0.237497 & 0.26 \\
\hline Rin2 & Down & 4 & 3.874568 & 0.002452 & 0.258093 & 0.25 \\
\hline Gem & Down & 3 & 3.882624 & 0.002302 & 0.257558 & 0.42735 \\
\hline Selenbp1 & Down & 3 & 4.462601 & 0.000293 & 0.224085 & 0.458333 \\
\hline Fcgrt & Down & 2 & 3.943613 & $3.45 E-05$ & 0.253575 & 0.5 \\
\hline Slc2a10 & Down & 2 & 3.864212 & $6.35 E-05$ & 0.258785 & 0.5 \\
\hline Syt12 & Down & 2 & 3.759494 & 0.000207 & 0.265993 & 0.5 \\
\hline Fam102a & Down & 1 & 5.730725 & 0 & 0.174498 & 0 \\
\hline Macrod1 & Down & 1 & 5.880322 & 0 & 0.170059 & 0 \\
\hline Pdgfrl & Down & 1 & 4.286536 & 0 & 0.233289 & 0 \\
\hline
\end{tabular}




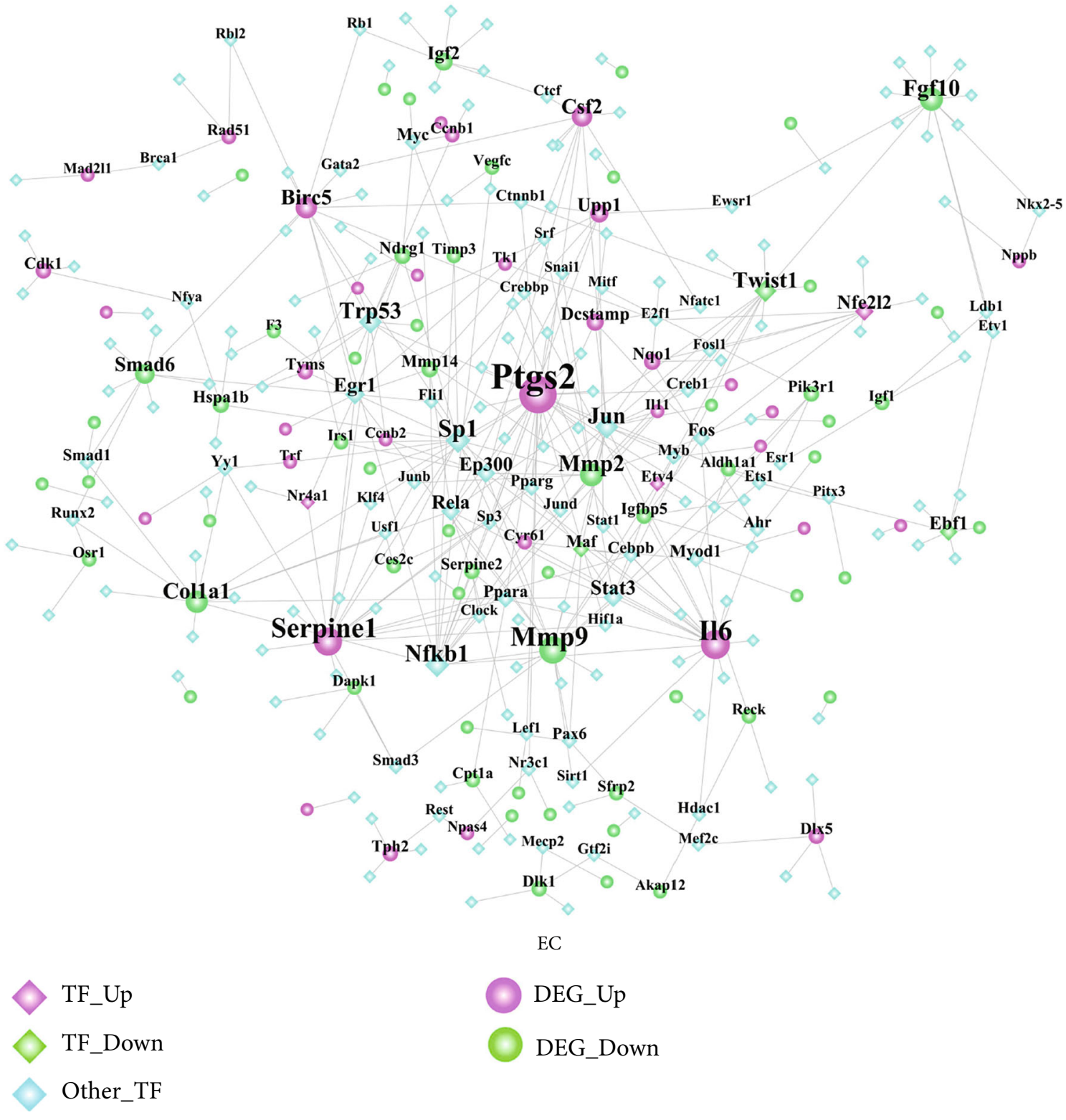

(a)

Figure 4: Continued. 


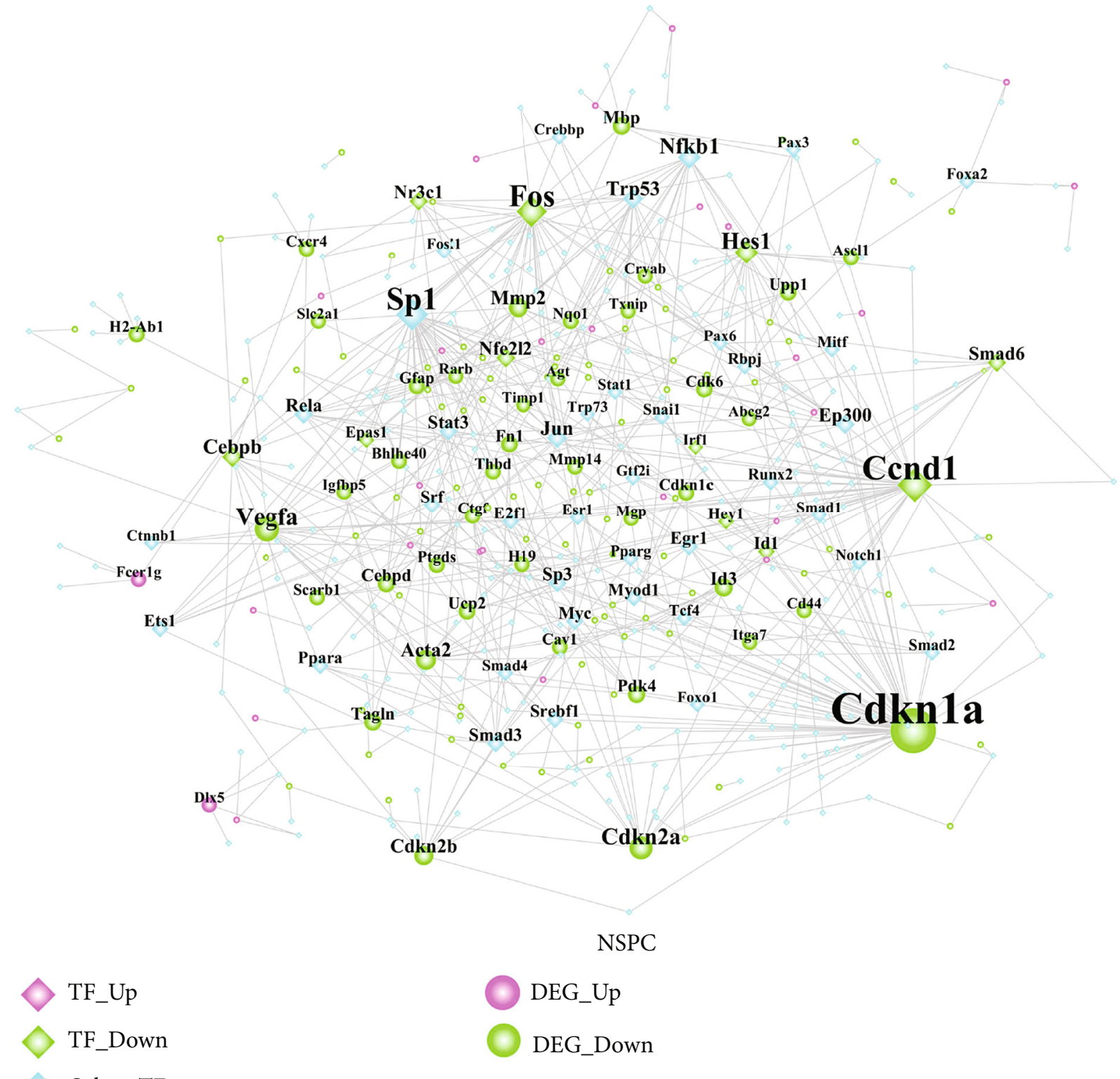

Other_TF

(b)

FIgURE 4: The transcription factor-target DEG interaction network. (a) The TF-target DEG network involved in the NSPC-induced EC alteration. (b) The TF-target DEG network involved in the EC-induced NSPC alteration.

11,675 edges (Figure 5(b)). The characteristics of the top 30 nodes of these two networks are listed in descending order of degree (Tables 11 and 12).

The miR-DEG-pathway network for three selected miRNAs (miR-210 (Figure 6(a)), miR-23a (Figure 6(b)), and miR-23b (Figure 6(c))) is depicted in Figure 6. As shown in Figure 6(a), miR-210 targets the Rhoq gene which was found to be downregulated in both NSPC-induced EC alteration and EC-induced NSPC alteration. The Rhoq gene was shown to be involved in the insulin signaling pathway. As shown in Figure 6(b), miR-23a targets the Aldh1a1 gene which was found to be downregulated in both NSPC-induced EC alteration and EC-induced NSPC alteration. The Aldh1al gene was shown to be involved in the retinol metabolism and met- abolic pathway. It could be observed from Figure 6(c) that the target genes of miR-23b were almost the same as those of miR-23a. miR-23b can target the Igf1 gene which was found to be downregulated in NSPC-induced EC alteration. The Igf1 gene was involved in many signaling pathways including AMPK, Rap1, HIF-1, p53, and FoxO signaling.

3.7. Identifying Significant Small Molecular Drugs. Figure 7 shows the DEmiRNA-small molecular drug network involved in the NSPC-induced EC alterations (Figure 7(a)) and EC-induced NSPC alterations (Figure 7(b)). Upon comparing the small molecules relevant to NSPC-induced EC alteration and EC-induced NSPC alteration, a total of 16 small drug molecules were found to be overlapped and 
TABLE 8: The topological characteristics of the top 30 nodes in the TF-target DEG interaction network involved in the NSPC-induced EC alteration.

\begin{tabular}{|c|c|c|c|c|c|c|}
\hline Name & Label & Degree & $\begin{array}{c}\text { Average shortest path } \\
\text { length }\end{array}$ & $\begin{array}{l}\text { Betweenness } \\
\text { centrality }\end{array}$ & $\begin{array}{l}\text { Closeness } \\
\text { centrality }\end{array}$ & $\begin{array}{c}\text { Topological } \\
\text { coefficient }\end{array}$ \\
\hline Ptgs2 & Upregulated DEG & 30 & 3.17073171 & 0.25370241 & 0.31538462 & 0.07348485 \\
\hline Il6 & Upregulated DEG & 20 & 3.31300813 & 0.16071429 & 0.30184049 & 0.10921053 \\
\hline Serpine1 & Upregulated DEG & 19 & 3.40650407 & 0.10638454 & 0.29355609 & 0.09078947 \\
\hline Mmp9 & Downregulated DEG & 18 & 3.25203252 & 0.16761464 & 0.3075 & 0.11243386 \\
\hline Sp1 & $\mathrm{TF}$ & 15 & 3.17479675 & 0.15105709 & 0.31498079 & 0.10813008 \\
\hline Nfkb1 & $\mathrm{TF}$ & 14 & 2.99593496 & 0.17606132 & 0.33378562 & 0.11316212 \\
\hline Mmp2 & Downregulated DEG & 13 & 3.45121951 & 0.05861543 & 0.28975265 & 0.16008316 \\
\hline Fgf10 & Downregulated DEG & 13 & 4.18699187 & 0.10945704 & 0.23883495 & 0.07692308 \\
\hline $\operatorname{Trp} 53$ & $\mathrm{TF}$ & 13 & 3.42276423 & 0.10326907 & 0.29216152 & 0.10697115 \\
\hline Jun & $\mathrm{TF}$ & 13 & 3.14634146 & 0.14436348 & 0.31782946 & 0.12725546 \\
\hline Twist1 & $\begin{array}{c}\text { Downregulated } \mathrm{DEG}_{-} \\
\mathrm{TF}\end{array}$ & 12 & 3.50406504 & 0.13470851 & 0.28538283 & 0.13288288 \\
\hline Colla1 & Downregulated DEG & 12 & 3.4796748 & 0.11936909 & 0.28738318 & 0.13709677 \\
\hline Birc5 & Upregulated DEG & 11 & 3.53252033 & 0.1098108 & 0.283084 & 0.13636364 \\
\hline Csf2 & Upregulated DEG & 10 & 3.51626016 & 0.08268292 & 0.28439306 & 0.16071429 \\
\hline Smad6 & Downregulated DEG & 9 & 4.94715447 & 0.04322026 & 0.2021364 & 0.12698413 \\
\hline Stat3 & $\mathrm{TF}$ & 9 & 3.43495935 & 0.09055949 & 0.29112426 & 0.15065913 \\
\hline Egr1 & $\mathrm{TF}$ & 9 & 3.76829268 & 0.05361397 & 0.26537217 & 0.13947991 \\
\hline $\mathrm{Nfe} 2 \mathrm{l} 2$ & Upregulated DEG_TF & 8 & 3.89430894 & 0.03472983 & 0.25678497 & 0.15972222 \\
\hline Rela & $\mathrm{TF}$ & 8 & 3.33333333 & 0.05841757 & 0.3 & 0.18382353 \\
\hline Ep300 & $\mathrm{TF}$ & 8 & 3.52439024 & 0.04168611 & 0.28373702 & 0.19067797 \\
\hline Upp1 & Upregulated DEG & 7 & 3.58536585 & 0.04680464 & 0.27891156 & 0.1978022 \\
\hline $\operatorname{Igf} 2$ & Downregulated DEG & 7 & 5.18292683 & 0.04071495 & 0.19294118 & 0.14285714 \\
\hline Ebf1 & $\begin{array}{c}\text { Downregulated } \mathrm{DEG}_{-} \\
\mathrm{TF}\end{array}$ & 7 & 4.57723577 & 0.04361263 & 0.21847247 & 0.14285714 \\
\hline Fos & $\mathrm{TF}$ & 6 & 3.82926829 & 0.01974273 & 0.2611465 & 0.22727273 \\
\hline Dcstamp & Upregulated DEG & 6 & 3.75203252 & 0.02000123 & 0.26652221 & 0.25396825 \\
\hline Nqo1 & Upregulated DEG & 5 & 3.83333333 & 0.02317522 & 0.26086957 & 0.21904762 \\
\hline Ndrg1 & Downregulated DEG & 5 & 3.83333333 & 0.02104419 & 0.26086957 & 0.27142857 \\
\hline Mmp14 & Downregulated DEG & 5 & 3.81707317 & 0.01378142 & 0.26198083 & 0.29 \\
\hline Maf & $\begin{array}{c}\text { Downregulated } \mathrm{DEG}_{-} \\
\mathrm{TF}\end{array}$ & 5 & 3.84146341 & 0.0340471 & 0.26031746 & 0.28571429 \\
\hline Hspalb & Downregulated DEG & 5 & 3.88211382 & 0.05662735 & 0.25759162 & 0.24545455 \\
\hline
\end{tabular}

significantly enriched (Table 13). Table 13 shows that 6 small molecular drugs (e.g., deptropine, fluphenazine, lycorine, quinostatin, resveratrol, and thiamazole) had similar regulatory roles in the process of EC-induced NSPC alteration and NSPC-induced EC alteration, indicating simultaneous upregulation or downregulation.

3.8. The miRNA-Environmental Chemical Compound Network. There were 60 environmental chemical compounds highly correlated with the DEmiRNAs expressed during NSPC-induced EC alteration and EC-induced NSPC alteration (Figure 8). As shown from Figure 8, miR-210 was significantly related to many compounds such as bisphenol A, titanium dioxide, choline, dietary fats, folic acid, and methionine. miR-23b was found to be significantly related to many compounds such as dexamethasone, potassium dichromate, sodium fluoride, thalidomide, cadmium chloride, and soot.

3.9. Crosstalk miRNAs Linking the EC-Induced NSPC Alteration Process and NSPC-Induced EC Alteration Process. A total of 54 interaction pairs of miRNAs were determined and are listed in Table 14. As shown in Figure 9, three miRNAs (miR-23a, miR-23b, and miR-210) were determined to perform critical bridging roles in the link between the ECinduced NSPC alteration process and the NSPC-induced EC alteration process. 
TABLE 9: The topological characteristics of the top 30 nodes in the TF-target DEG interaction network involved in the EC-induced NSPC alteration.

\begin{tabular}{|c|c|c|c|c|c|c|}
\hline Name & Label & Degree & $\begin{array}{c}\text { Average shortest path } \\
\text { length }\end{array}$ & $\begin{array}{c}\text { Betweenness } \\
\text { centrality }\end{array}$ & $\begin{array}{l}\text { Closeness } \\
\text { centrality }\end{array}$ & $\begin{array}{c}\text { Topological } \\
\text { coefficient }\end{array}$ \\
\hline Cdknla & Downregulated DEG & 65 & 2.73913043 & 0.30858346 & 0.36507937 & 0.0283698 \\
\hline Ccnd1 & $\begin{array}{c}\text { Downregulated } \mathrm{DEG}_{-} \\
\mathrm{TF}\end{array}$ & 44 & 2.90096618 & 0.18721585 & 0.34471274 & 0.0390556 \\
\hline Sp1 & $\mathrm{TF}$ & 38 & 2.75120773 & 0.18665254 & 0.36347673 & 0.04086687 \\
\hline Fos & $\begin{array}{c}\text { Downregulated } \mathrm{DEG}_{-} \\
\mathrm{TF}\end{array}$ & 37 & 2.89855072 & 0.15489676 & 0.345 & 0.05012705 \\
\hline Vegfa & Downregulated DEG & 24 & 3.09178744 & 0.1032236 & 0.3234375 & 0.06904762 \\
\hline Hes 1 & $\begin{array}{c}\text { Downregulated } \text { DEG }_{-} \\
\text {TF }\end{array}$ & 21 & 3.26328502 & 0.093159 & 0.30643967 & 0.06776557 \\
\hline Cdkn2a & Downregulated DEG & 20 & 3.16183575 & 0.06806015 & 0.31627196 & 0.0622093 \\
\hline Nfkb1 & $\mathrm{TF}$ & 19 & 2.83091787 & 0.10896078 & 0.35324232 & 0.078356 \\
\hline Cebpb & $\begin{array}{c}\text { Downregulated } \mathrm{DEG}_{-} \\
\mathrm{TF}\end{array}$ & 15 & 3.01690821 & 0.0630859 & 0.33146517 & 0.07979003 \\
\hline Acta2 & Downregulated DEG & 14 & 3.41062802 & 0.04841393 & 0.29320113 & 0.10449735 \\
\hline Trp53 & $\mathrm{TF}$ & 14 & 3.23188406 & 0.04815141 & 0.30941704 & 0.09895151 \\
\hline Jun & $\mathrm{TF}$ & 14 & 3.25603865 & 0.03607564 & 0.30712166 & 0.10204082 \\
\hline Mmp2 & Downregulated DEG & 13 & 3.08937198 & 0.03126342 & 0.32369038 & 0.11609687 \\
\hline Ep300 & $\mathrm{TF}$ & 12 & 3.13043478 & 0.05353532 & 0.31944444 & 0.11131841 \\
\hline $\mathrm{Cdkn} 2 \mathrm{~b}$ & Downregulated DEG & 12 & 3.80917874 & 0.03041314 & 0.26252378 & 0.10714286 \\
\hline $\mathrm{Nfe} 2 \mathrm{l} 2$ & $\begin{array}{c}\text { Downregulated } \mathrm{DEG}_{-} \\
\mathrm{TF}\end{array}$ & 12 & 3.26570048 & 0.04141463 & 0.30621302 & 0.10585586 \\
\hline Rela & $\mathrm{TF}$ & 11 & 3.21497585 & 0.03799736 & 0.31104433 & 0.118411 \\
\hline $\mathrm{Nr} 3 \mathrm{cl}$ & $\begin{array}{c}\text { Downregulated } \text { DEG }_{-} \\
\text {TF }\end{array}$ & 11 & 3.24637681 & 0.03066868 & 0.30803571 & 0.11162255 \\
\hline Smad6 & $\begin{array}{c}\text { Downregulated } \mathrm{DEG}_{-} \\
\mathrm{TF}\end{array}$ & 11 & 3.73671498 & 0.02413405 & 0.26761474 & 0.11004785 \\
\hline Sp3 & $\mathrm{TF}$ & 10 & 3.35990338 & 0.01668852 & 0.29762761 & 0.12268041 \\
\hline Stat3 & $\mathrm{TF}$ & 10 & 3.33816425 & 0.03841092 & 0.29956585 & 0.13235294 \\
\hline Id3 & Downregulated DEG & 10 & 3.82125604 & 0.02673839 & 0.26169406 & 0.10869565 \\
\hline Smad3 & $\mathrm{TF}$ & 9 & 3.72463768 & 0.03330304 & 0.26848249 & 0.14403292 \\
\hline Egr1 & $\mathrm{TF}$ & 9 & 3.57004831 & 0.03896833 & 0.28010825 & 0.12007168 \\
\hline Mbp & Downregulated DEG & 9 & 3.52415459 & 0.02687184 & 0.283756 & 0.13888889 \\
\hline Srebf1 & $\mathrm{TF}$ & 8 & 3.59903382 & 0.0307691 & 0.27785235 & 0.13380282 \\
\hline Myc & $\mathrm{TF}$ & 8 & 3.36231884 & 0.02336862 & 0.29741379 & 0.14650538 \\
\hline Id1 & $\begin{array}{c}\text { Downregulated } \mathrm{DEG}_{-} \\
\mathrm{TF}\end{array}$ & 8 & 3.2826087 & 0.02185908 & 0.30463576 & 0.13903061 \\
\hline Cebpd & Downregulated DEG & 8 & 3.43236715 & 0.01679448 & 0.29134412 & 0.16163793 \\
\hline Pdk4 & Downregulated DEG & 8 & 3.61111111 & 0.01282401 & 0.27692308 & 0.19396552 \\
\hline
\end{tabular}

TABLE 10: The number of experimentally validated, computationally predicted, and total miRNA-target DEG interaction pairs during the NSPC-induced EC alteration and EC-induced NSPC alteration.

\begin{tabular}{|c|c|c|c|c|}
\hline $\begin{array}{l}\text { Coculture-induced } \\
\text { alterations in one } \\
\text { type of cells }\end{array}$ & $\begin{array}{l}\text { Validated } \\
\text { miRNA-target } \\
\text { DEG pairs }\end{array}$ & $\begin{array}{l}\text { Predicted } \\
\text { miRNA-target } \\
\text { DEG pairs }\end{array}$ & $\begin{array}{l}\text { Total miRNA-target DEG } \\
\text { pairs (including validated } \\
\quad \text { and predicted) }\end{array}$ & $\begin{array}{l}\text { Overlapped miRNA-target DEG interaction pairs (overlapped } \\
\text { between NSPC-induced EC alteration and EC-induced NSPC } \\
\text { alteration) }\end{array}$ \\
\hline $\begin{array}{l}\text { NSPC-induced EC } \\
\text { alteration }\end{array}$ & 536 & 391 & 810 & $\begin{array}{l}\text { The overlapped } 14 \text { interaction pairs are as follows: miR-21-Rhoq, } \\
\text { miR-23a-Akap12, miR-23a-Aldh1a1, miR-23a-Exoc314, miR-23a- }\end{array}$ \\
\hline $\begin{array}{l}\text { EC-induced NSPC } \\
\text { alteration }\end{array}$ & 571 & 243 & 711 & $\begin{array}{l}\text { Gm5424, miR-23a-Mfhas1, miR-23a-Nfe2l2, miR-23a-Slc16a6, } \\
\text { miR-23b-Akap12, miR-23b-Aldh1a1, miR-23b-Gm5424, miR-23b- } \\
\text { Mfhas1, miR-23b-Nfe212, and miR-23b-Slc16a6. }\end{array}$ \\
\hline
\end{tabular}




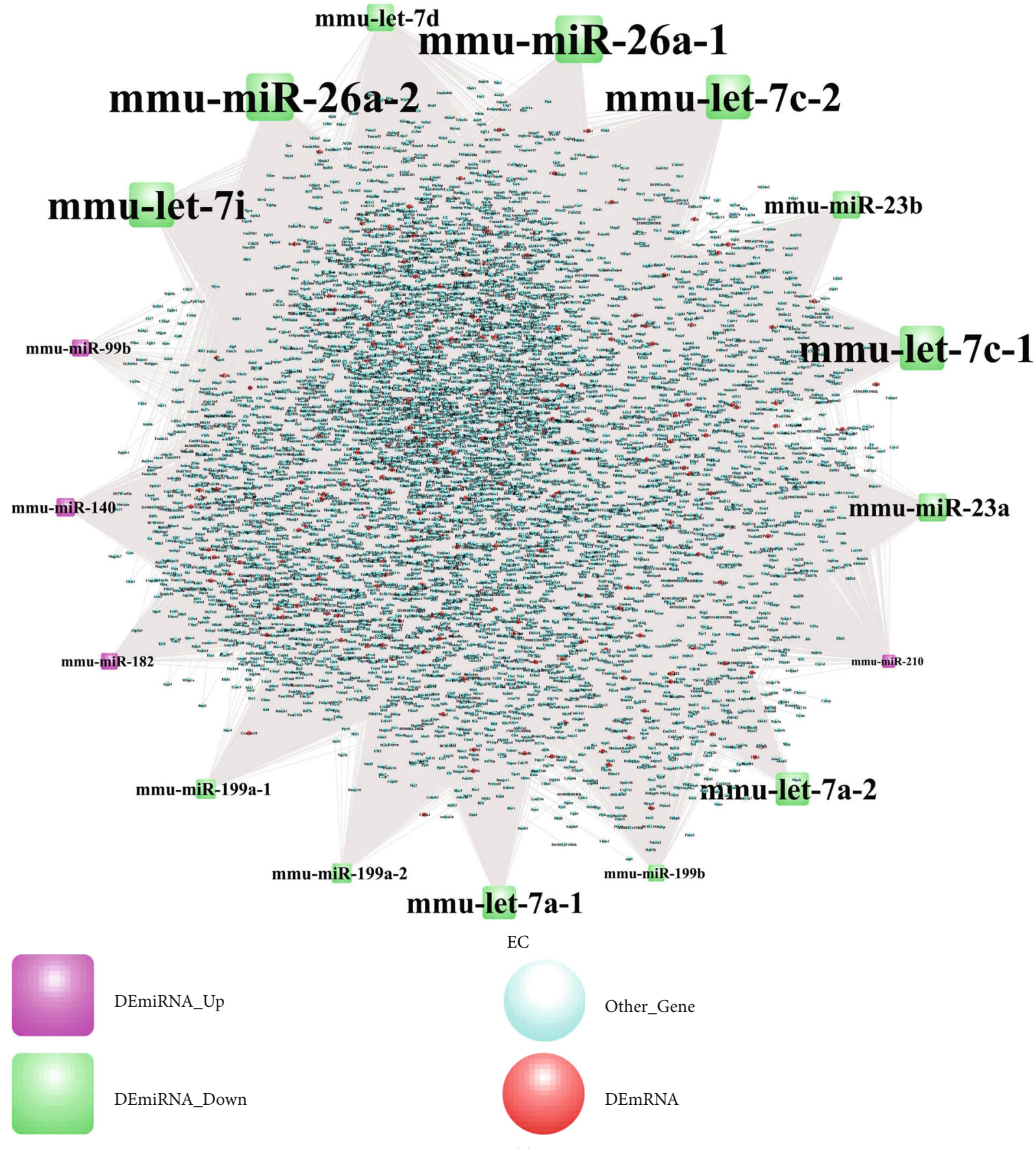

(a)

Figure 5: Continued. 


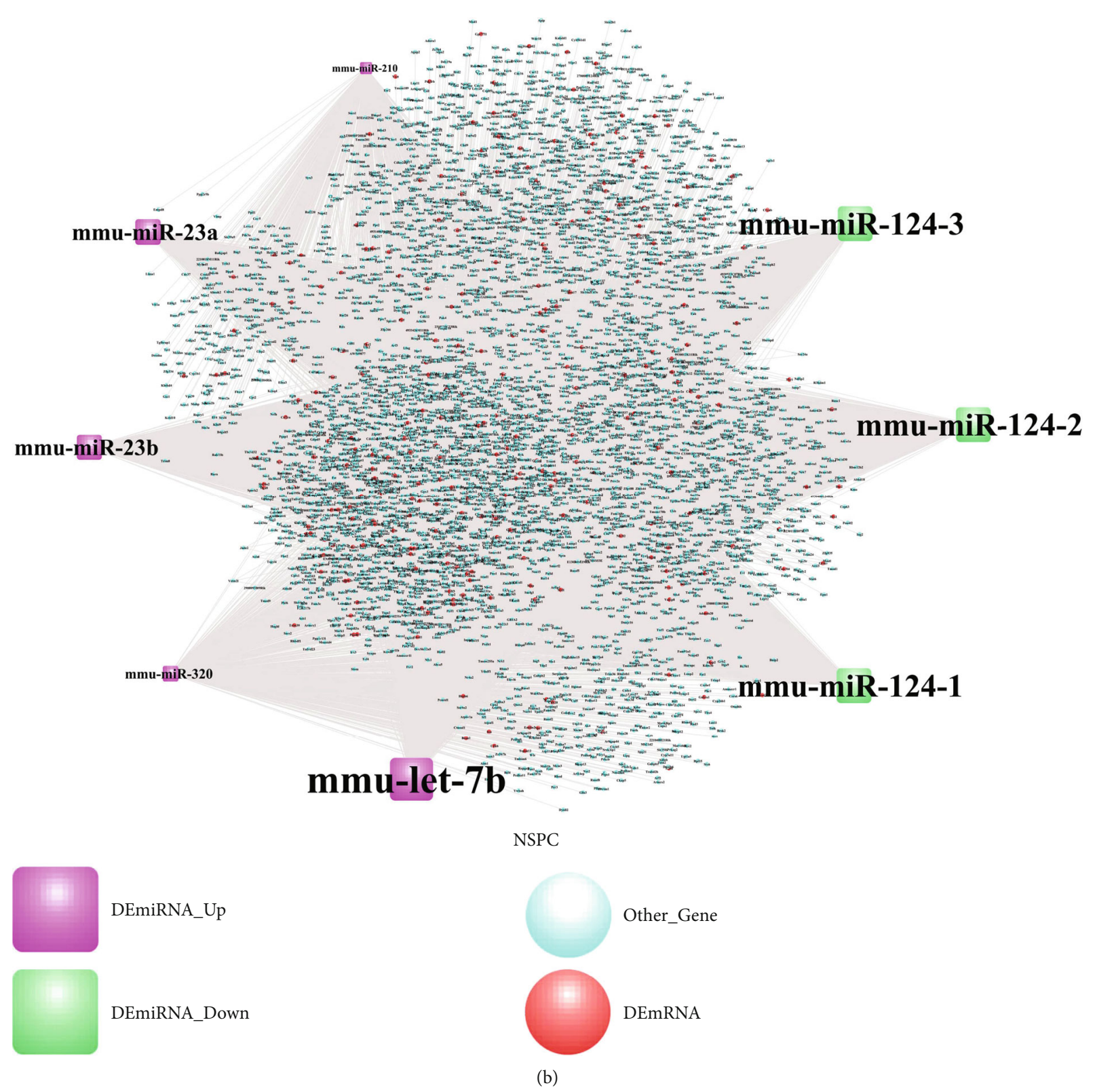

FIGURE 5: The DEmiRNA-target network involved in the NSPC-induced EC alteration (a) and EC-induced NSPC alteration (b).

\section{Discussion}

This study identified three miRNAs (i.e., miR-210, miR-230a, and miR-23b) to be crosstalks involved in the interaction between ECs and NSPCs. The roles of these miRNAs in regulating the interactions between ECs and NSPCs will be discussed in the following sections by using the previous related evidence.

miR-210 is identified to be involved in the interaction between ECs and NSPCs by targeting many genes (e.g., Rhoq, Fn1, and Pard3) and pathways (e.g., insulin, PI3K/Akt, and chemokine signaling pathway) (Figure 3(a)), and its regulating roles will be discussed in this and next paragraph. Rhoq (Ras Homolog (Rho) Family Member Q) was identified to be downregulated during the process of EC-induced NSPC alteration and NSPC-induced EC alteration; however, Rhoq has not been investigated to regulate NSPCs and ECs. As another member of the Rho family, RhoA was found to influence the proliferation and fate of NSPCs by mediating mechanotransduction and cellular stiffness [29, 30], as well as impact the migration and angiogenesis of ECs by mediating the cytoskeletal changes [31]. The insulin signaling pathway targeted by RhoA could promote angiogenic processes via mediating the migration and proliferation of ECs as well as in vitro tubular structure formation [32]. The insulinmediated pathway could also regulate the self-renewal, neurogenesis, cognition, and sensory function, as well as homeostasis of NSPCs [33]. 
TABLE 11: The topological characteristics of the top 30 nodes in the DEmiRNA-target network involved in the NSPC-induced EC alteration.

\begin{tabular}{|c|c|c|c|c|c|c|}
\hline Name & lab2 & Degree & $\begin{array}{c}\text { Average shortest path } \\
\text { length }\end{array}$ & $\begin{array}{l}\text { Betweenness } \\
\text { centrality }\end{array}$ & $\begin{array}{l}\text { Closeness } \\
\text { centrality }\end{array}$ & $\begin{array}{c}\text { Topological } \\
\text { coefficient }\end{array}$ \\
\hline mmu-miR-26a-2 & DEmiRNA & 2371 & 2.195604 & 0.216585 & 0.455455 & 0.221505 \\
\hline mmu-miR-26a-1 & DEmiRNA & 2371 & 2.195604 & 0.216585 & 0.455455 & 0.221505 \\
\hline mmu-let-7i & DEmiRNA & 2244 & 2.238546 & 0.131513 & 0.446719 & 0.323641 \\
\hline mmu-let-7c-2 & DEmiRNA & 2202 & 2.252747 & 0.110037 & 0.443902 & 0.332539 \\
\hline mmu-let-7c-1 & DEmiRNA & 2202 & 2.252747 & 0.110037 & 0.443902 & 0.332539 \\
\hline mmu-let-7a-2 & DEmiRNA & 1585 & 2.461369 & 0.051855 & 0.406278 & 0.376735 \\
\hline mmu-let-7a-1 & DEmiRNA & 1585 & 2.461369 & 0.051855 & 0.406278 & 0.376735 \\
\hline mmu-miR-23a & DEmiRNA & 1297 & 2.558749 & 0.106936 & 0.390816 & 0.247494 \\
\hline mmu-miR-23b & DEmiRNA & 1269 & 2.568216 & 0.100185 & 0.389375 & 0.248916 \\
\hline mmu-let-7d & DEmiRNA & 1252 & 2.573965 & 0.063356 & 0.388506 & 0.353484 \\
\hline $\begin{array}{l}\text { mmu-miR-199a- } \\
2\end{array}$ & DEmiRNA & 839 & 2.713609 & 0.055721 & 0.368513 & 0.273838 \\
\hline $\begin{array}{l}\text { mmu-miR-199a- } \\
1\end{array}$ & DEmiRNA & 839 & 2.713609 & 0.055721 & 0.368513 & 0.273838 \\
\hline mmu-miR-140 & DEmiRNA & 760 & 2.740321 & 0.114496 & 0.364921 & 0.175411 \\
\hline mmu-miR-182 & DEmiRNA & 668 & 2.771429 & 0.088383 & 0.360825 & 0.194891 \\
\hline mmu-miR-199b & DEmiRNA & 660 & 2.774134 & 0.048537 & 0.360473 & 0.278883 \\
\hline mmu-miR-210 & DEmiRNA & 468 & 2.839053 & 0.061318 & 0.35223 & 0.195913 \\
\hline mmu-miR-99b & DEmiRNA & 52 & 2.979713 & 0.006853 & 0.335603 & 0.234375 \\
\hline Celf1 & $\begin{array}{l}\text { Target } \\
\text { gene }\end{array}$ & 16 & 2.004227 & 0.002733 & 0.498946 & 0.24026 \\
\hline Taok1 & $\begin{array}{l}\text { Target } \\
\text { gene }\end{array}$ & 15 & 2.179374 & 0.001948 & 0.458847 & 0.263894 \\
\hline Ubn2 & $\begin{array}{l}\text { Target } \\
\text { gene }\end{array}$ & 14 & 2.137447 & 0.001613 & 0.467848 & 0.279439 \\
\hline Suco & $\begin{array}{l}\text { Target } \\
\text { gene }\end{array}$ & 14 & 2.137447 & 0.001613 & 0.467848 & 0.279439 \\
\hline $\mathrm{Mtf} 2$ & $\begin{array}{l}\text { Target } \\
\text { gene }\end{array}$ & 14 & 2.186475 & 0.001497 & 0.457357 & 0.28312 \\
\hline Map3k2 & $\begin{array}{l}\text { Target } \\
\text { gene }\end{array}$ & 14 & 2.162468 & 0.001485 & 0.462435 & 0.282045 \\
\hline Kdm6a & $\begin{array}{l}\text { Target } \\
\text { gene }\end{array}$ & 14 & 2.162468 & 0.001485 & 0.462435 & 0.282045 \\
\hline Ino80d & $\begin{array}{l}\text { Target } \\
\text { gene }\end{array}$ & 14 & 2.137109 & 0.001824 & 0.467922 & 0.267984 \\
\hline Celf2 & $\begin{array}{l}\text { Target } \\
\text { gene }\end{array}$ & 14 & 2.090786 & 0.001937 & 0.478289 & 0.265165 \\
\hline Ankrd44 & $\begin{array}{l}\text { Target } \\
\text { gene }\end{array}$ & 14 & 2.186475 & 0.001497 & 0.457357 & 0.28312 \\
\hline Tnpol & $\begin{array}{l}\text { Target } \\
\text { gene }\end{array}$ & 13 & 2.196281 & 0.001334 & 0.455315 & 0.291288 \\
\hline $\mathrm{Rfx} 7$ & $\begin{array}{l}\text { Target } \\
\text { gene }\end{array}$ & 13 & 2.144548 & 0.001367 & 0.466299 & 0.28419 \\
\hline $\mathrm{Kmt} 2 \mathrm{a}$ & $\begin{array}{l}\text { Target } \\
\text { gene }\end{array}$ & 13 & 2.263567 & 0.001442 & 0.441781 & 0.289898 \\
\hline
\end{tabular}

During the interactions between ECs and NSPCs, the genes dysregulated by a certain type of cell might influence the biological behavior and processes of another type of cell. For instance, the addition of ECs into NSPCs induced the downregulation of the gene Fn1 (fibronectin-1) in NSPCs. While fibronectin was shown to regulate the survival and migration of primary NSPCs [34], its dysregulation in NSPCs can modulate the functions of ECs by influencing survival 
TABLE 12: The topological characteristics of the top 30 nodes in the DEmiRNA-target network involved in the EC-induced NSPC alteration.

\begin{tabular}{|c|c|c|c|c|c|c|}
\hline Name & lab2 & Degree & $\begin{array}{c}\text { Average shortest path } \\
\text { length }\end{array}$ & $\begin{array}{l}\text { Betweenness } \\
\text { centrality }\end{array}$ & $\begin{array}{l}\text { Closeness } \\
\text { centrality }\end{array}$ & $\begin{array}{c}\text { Topological } \\
\text { coefficient }\end{array}$ \\
\hline mmu-let-7b & DEmiRNA_up & 2362 & 2.046362 & 0.551173 & 0.488672 & 0.170618 \\
\hline $\begin{array}{l}\text { mmu-miR-124- } \\
1\end{array}$ & $\begin{array}{c}\text { DEmiRNA_ } \\
\text { down }\end{array}$ & 1863 & 2.247531 & 0.154513 & 0.444933 & 0.394448 \\
\hline $\begin{array}{l}\text { mmu-miR-124- } \\
2\end{array}$ & $\begin{array}{c}\text { DEmiRNA_ } \\
\text { down }\end{array}$ & 1863 & 2.247531 & 0.154513 & 0.444933 & 0.394448 \\
\hline $\begin{array}{l}\text { mmu-miR-124- } \\
3\end{array}$ & $\begin{array}{c}\text { DEmiRNA_ } \\
\text { down }\end{array}$ & 1863 & 2.247531 & 0.154513 & 0.444933 & 0.394448 \\
\hline mmu-miR-23a & DEmiRNA_up & 1297 & 2.475711 & 0.158061 & 0.403924 & 0.306091 \\
\hline mmu-miR-23b & DEmiRNA_up & 1269 & 2.486999 & 0.14813 & 0.402091 & 0.309242 \\
\hline mmu-miR-320 & DEmiRNA_up & 690 & 2.720419 & 0.141572 & 0.36759 & 0.217805 \\
\hline mmu-miR-210 & DEmiRNA_up & 468 & 2.809917 & 0.093463 & 0.355882 & 0.206654 \\
\hline Ino80d & Target gene & 8 & 1.998387 & 0.0024 & 0.500403 & 0.294443 \\
\hline Adam 10 & Target gene & 8 & 1.998387 & 0.0024 & 0.500403 & 0.294443 \\
\hline Mtf2 & Target gene & 8 & 1.998387 & 0.0024 & 0.500403 & 0.294443 \\
\hline Pcdh19 & DEmRNA & 7 & 2.085467 & 0.001524 & 0.479509 & 0.337695 \\
\hline Rora & DEmRNA & 7 & 2.545051 & 0.001403 & 0.392919 & 0.369491 \\
\hline Ago2 & Target gene & 7 & 2.1282 & 0.00156 & 0.469881 & 0.338576 \\
\hline Ago3 & Target gene & 7 & 2.1282 & 0.00156 & 0.469881 & 0.338576 \\
\hline Col4a1 & Target gene & 7 & 2.085467 & 0.001524 & 0.479509 & 0.337695 \\
\hline Cpd & Target gene & 7 & 2.085467 & 0.001524 & 0.479509 & 0.337695 \\
\hline Map3k1 & Target gene & 7 & 2.085467 & 0.001524 & 0.479509 & 0.337695 \\
\hline Mecp2 & Target gene & 7 & 2.085467 & 0.001524 & 0.479509 & 0.337695 \\
\hline Etnk1 & Target gene & 7 & 2.085467 & 0.001524 & 0.479509 & 0.337695 \\
\hline Kpna1 & Target gene & 7 & 2.085467 & 0.001524 & 0.479509 & 0.337695 \\
\hline Yod1 & Target gene & 7 & 2.085467 & 0.001524 & 0.479509 & 0.337695 \\
\hline Prtg & Target gene & 7 & 2.085467 & 0.001524 & 0.479509 & 0.337695 \\
\hline Slc36a1 & Target gene & 7 & 2.1282 & 0.00156 & 0.469881 & 0.338576 \\
\hline Tet2 & Target gene & 7 & 2.1282 & 0.00156 & 0.469881 & 0.338576 \\
\hline Ahctf1 & Target gene & 7 & 2.1282 & 0.00156 & 0.469881 & 0.338576 \\
\hline Qser1 & Target gene & 7 & 2.085467 & 0.001524 & 0.479509 & 0.337695 \\
\hline Wnk1 & Target gene & 7 & 2.085467 & 0.001524 & 0.479509 & 0.337695 \\
\hline Slc7a2 & Target gene & 7 & 2.085467 & 0.001524 & 0.479509 & 0.337695 \\
\hline Aldh 112 & Target gene & 7 & 2.545051 & 0.001403 & 0.392919 & 0.369491 \\
\hline
\end{tabular}

and angiogenesis in both the integrin-dependent and integrin-independent manners [35]. The PI3K/Akt pathway targeted by Fn1 has been established as a classic pathway in regulating both angiogenesis and neurogenesis. This pathway plays a mediating role in neural regeneration by controlling the survival, proliferation, differentiation, and migration of NSPCs [36]. The link between this pathway and angiogenesis has been highlighted since it can modulate the expression of many angiogenic factors such as vascular endothelial growth factor (VEGF), nitric oxide, and angiopoietins [37]. As another example, the addition of NSPCs to ECs induced a decreased expression of the gene Pard3 (Par-3 Family Cell Polarity Regulator) in ECs. Pard3 can not only determine the cell polarity of ECs [38] but also modulate the sprouting behavior of ECs during the process of angiogenesis [39]. Conversely, the dysregulation of cell polarity regulators can influence the behavior and functions of NSPCs in terms of architecture and shape, interkinetic nuclear migration, proliferation and differentiation potential, and asymmetric cell division [40]. The chemokine signaling pathway targeted by Pard3 was shown to promote neovascularization by stimulating the migration and proliferation of ECs, as well as recruiting endothelial progenitor cells [41]. Regarding the role of chemokine in regulating NSPCs, chemokines were shown to promote the quiescence and survival of NSPCs [42], as well as regulate the migration of NSPCs toward the sites of neuroinflammation [43]. Overall, based on this existing evidence, it can be assumed that miR-210 plays a critical role in the interaction between ECs and NSPCs by targeting genes and pathways associated with both neurogenesis and angiogenesis. 


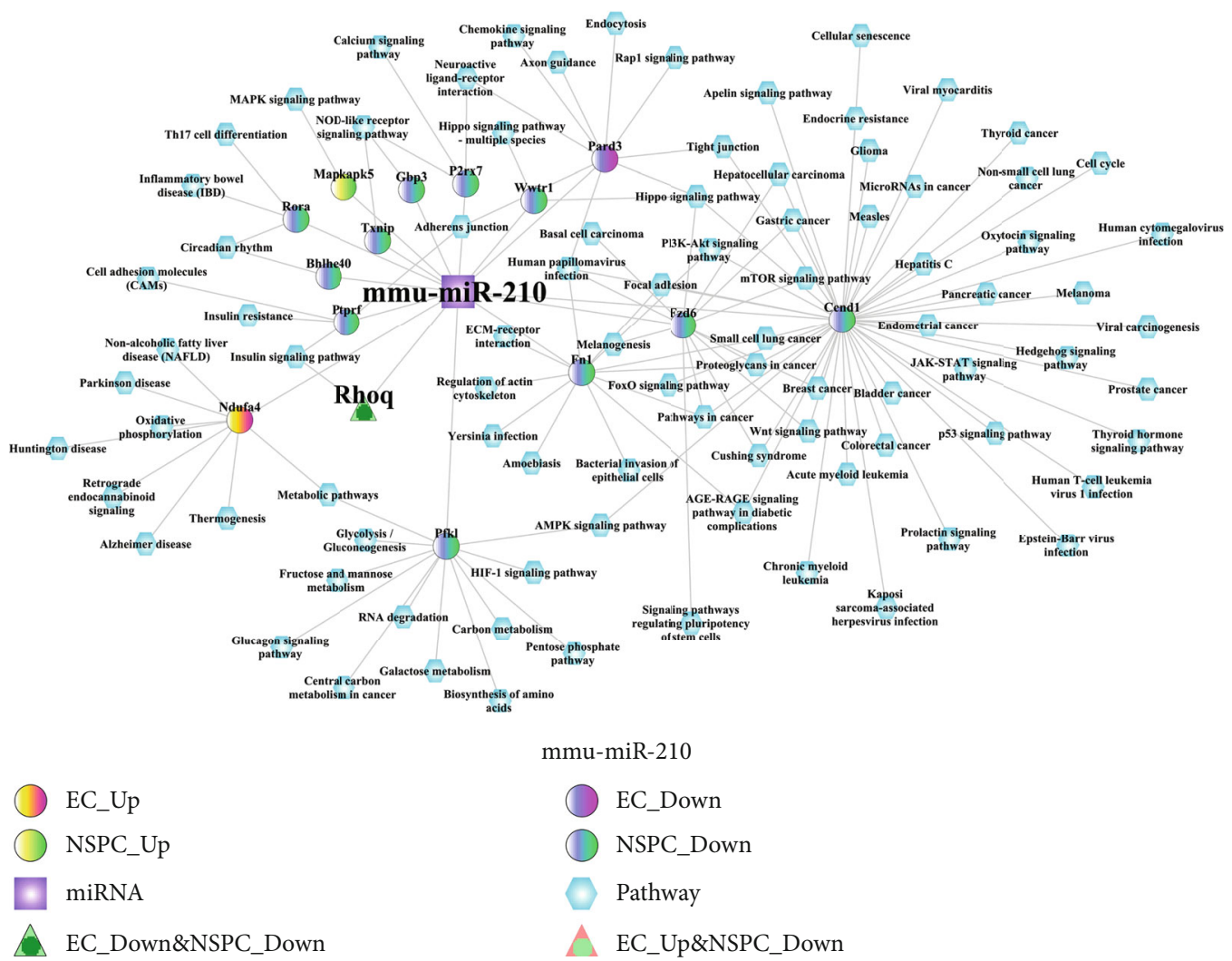

(a)

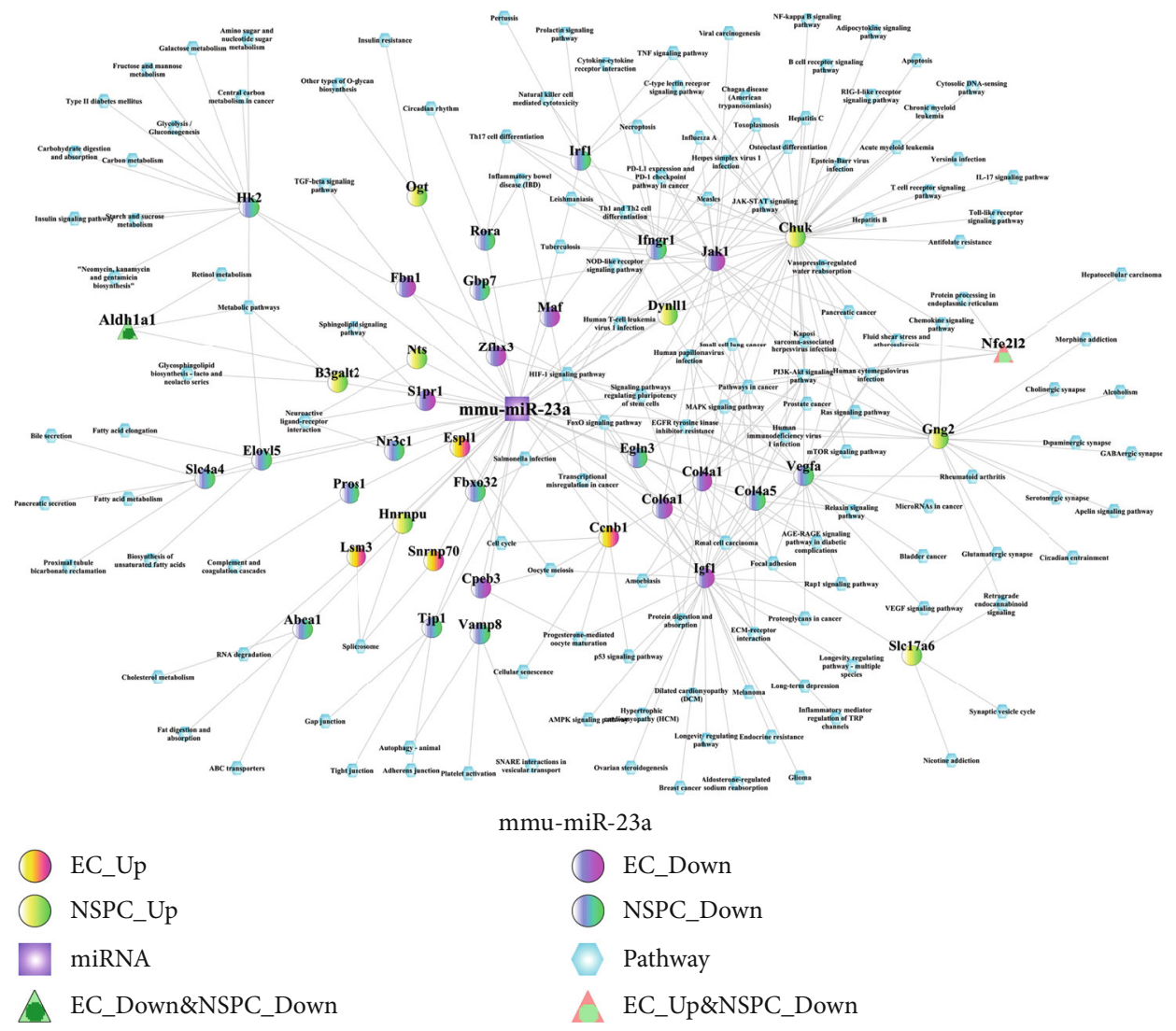

(b)

Figure 6: Continued. 


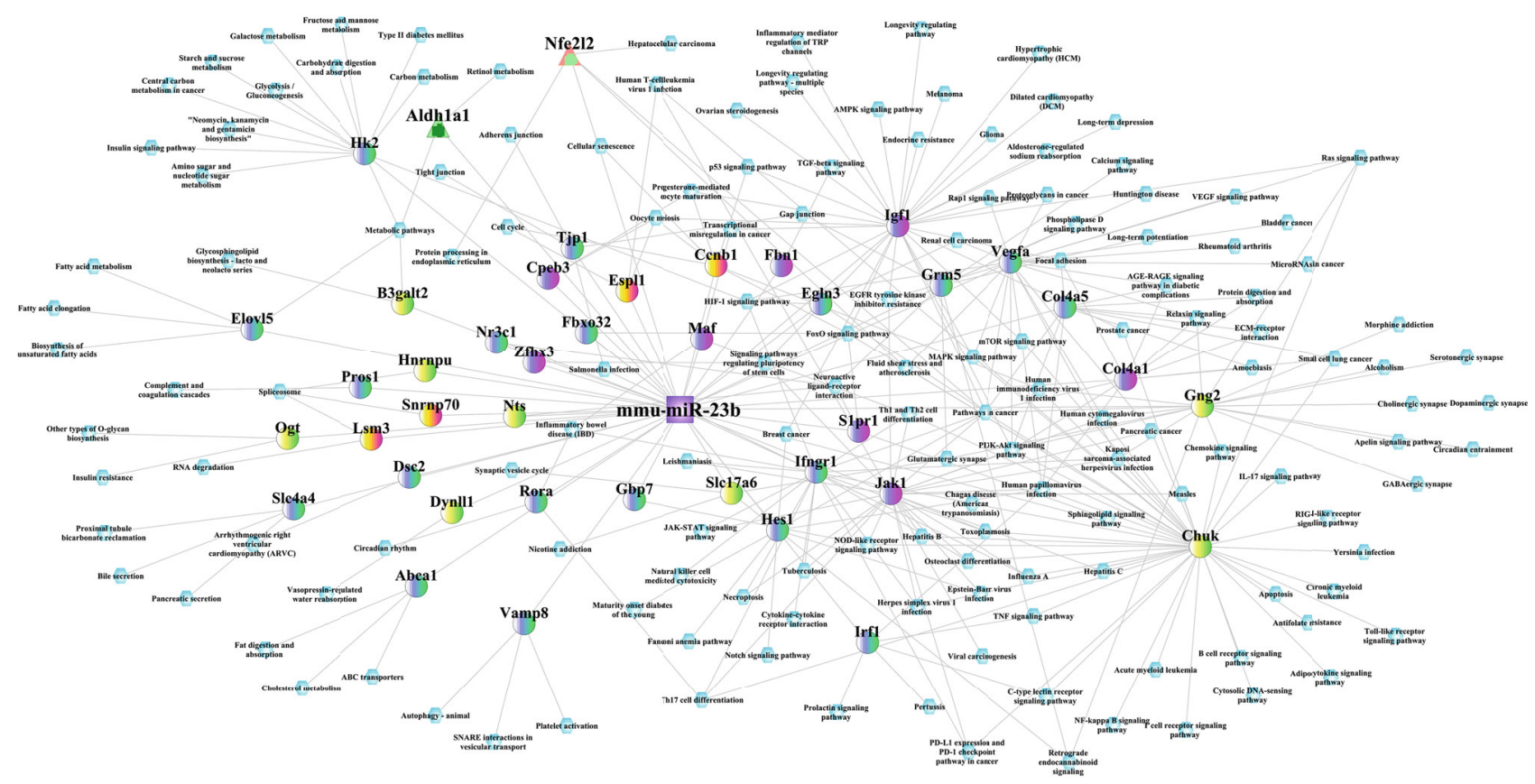

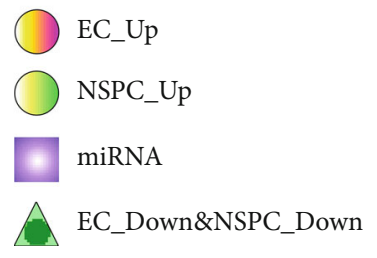

FIgure 6: The miRNA-DEG-pathway interaction network.
EC_Down

NSPC_Down

Pathway

EC_Up\&NSPC_Down

(c)
The mechanisms of two miRNAs (miR-23a and miR$23 \mathrm{~b}$ ) involved in the interaction between ECs and NSPCs are almost similar, and the regulatory roles of miR-23a will be therefore an example to discuss in this and next paragraph. As shown in Figure 3(b), miR-23a is involved in the interaction between ECs and NSPCs by targeting many genes (e.g., Aldh1a1, Fbn1, and Chuk) and pathways (e.g., retinol metabolism, TGF-beta, and NF- $\kappa$ B pathway). For example, the Aldh1a1 (Aldehyde Dehydrogenase 1 Family Member A1) gene targeted by miR-23a was found to be simultaneously downregulated during the process of EC-induced NSPC alteration and NSPC-induced EC alteration. High activity of aldehyde dehydrogenase (ALDH) in tumor ECs and its function in promoting angiogenesis in tumor progression have been reported [44]; however, so far, there is no evidence supporting the angiogenic role of ALDH in stem celllike ECs. A recent review [45] reported that aldehyde dehydrogenase $(\mathrm{ALDH})$ not only represents an intracellular and metabolic marker of stem cells including NSPCs and endothelial progenitor cells (EPCs) but also acts as a functional regulator of stem cells mainly by mediating the metabolic pathway of retinoic acid (RA). The retinol metabolism pathway targeted by Aldh1a1 was shown to be involved at the early stage of neurogenesis and antiangiogenic response. In terms of neurogenesis, RA has been commonly applied for differentiating NSPCs due to its function in promoting the acquisition of a neuronal fate [46]. In terms of antiangiogenic response, RA has been shown to selectively block vascular permeability factor/vascular endothelial growth factor (VPF/VEGF), thereby further inhibiting these angiogenic factors that induced microvascular permeability [47].

The interaction of ECs and NSPCs supports the angiogenesis of ECs by influencing the expression of angiogenesis-related genes in ECs; likewise, this interaction also supports the neurogenesis of NSPCs by impacting the neurogenesis-associated genes in NSPCs. For instance, the addition of NSPCs into ECs resulted in the downregulation of Fbn1 (Fibrillin 1) in ECs. The encoded protein of gene Fbn1 is a large extracellular matrix (ECM) glycoprotein, which assembles to form $10-12 \mathrm{~nm}$ microfibrils in ECM. ECM has been demonstrated as a hard player in regulating angiogenesis via interacting/restoring a variety of growth factors (e.g., fibroblast growth factor (FGF), platelet-derived growth factor (PDGF), hepatocyte growth factor (HGF), and VEGF) [48]. In addition to its involvement in angiogenesis, ECM was shown to regulate the proliferation and differentiation of NSPCs by inducing the local alterations in the substrate stiffness [30]. The TGF-beta signaling pathway targeted by Fbn 1 has been regarded as an indispensable player in facilitating interactions between ECs and NSPCs based on its function in vasculogenesis and angiogenesis by upregulating the expression of angiogenic factors [49], as well as its function in neurogenesis by modulating the temporal specification and progenitor potency of NSPCs [50]. As another 


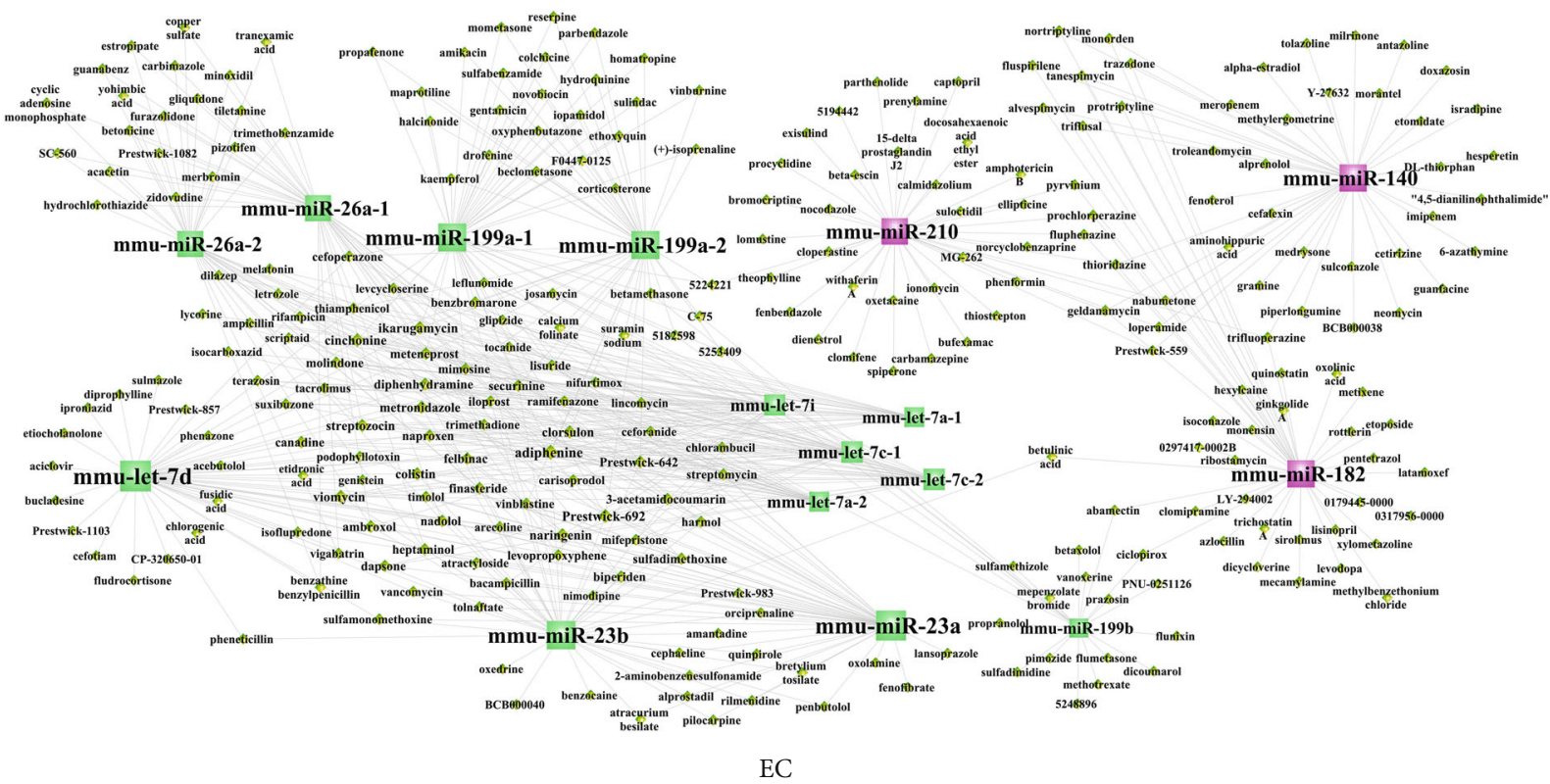

DEmiRN_Up

DEmiRNA_Down

Enrichment

(a)

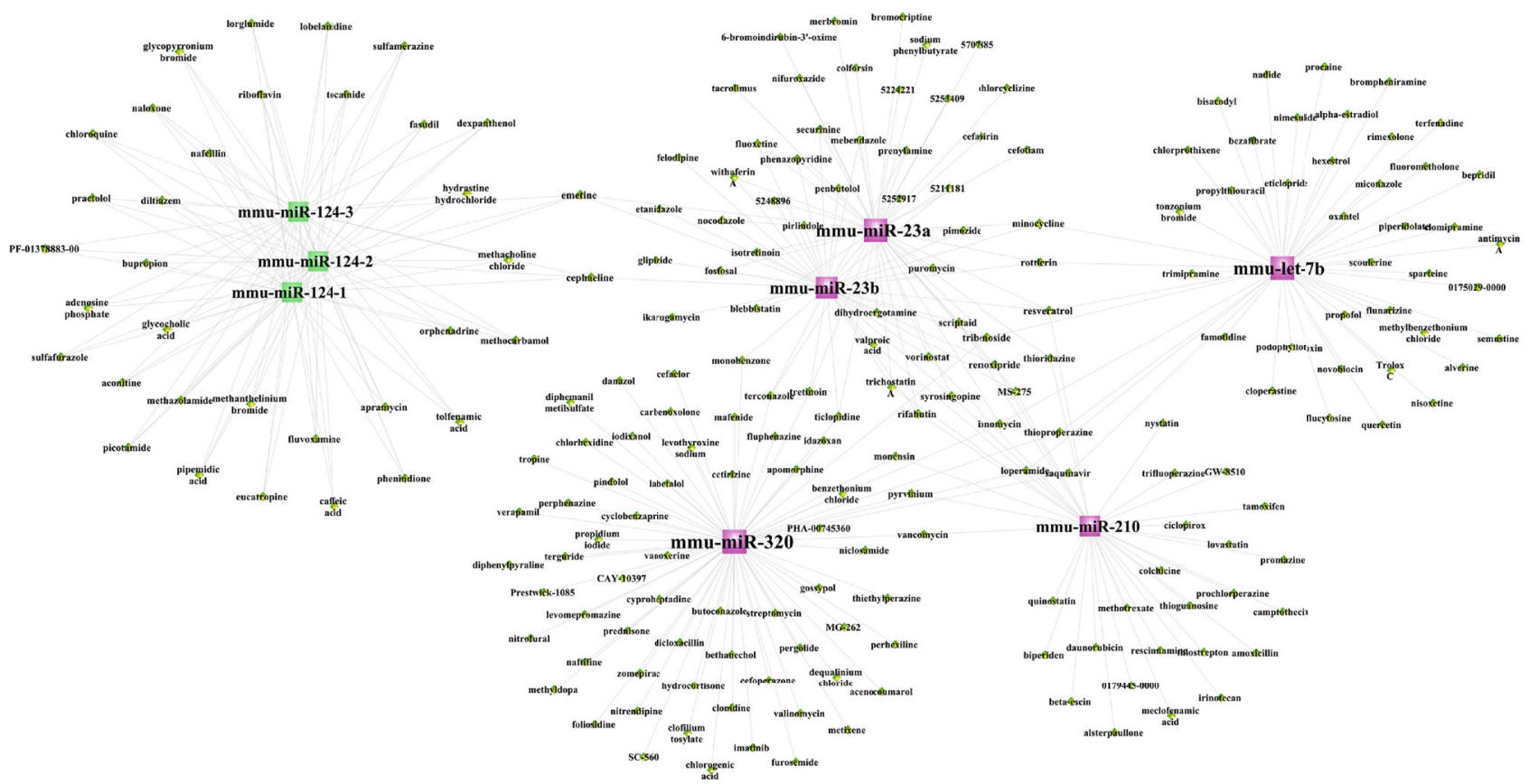

DEmiRN_Up

DEmiRNA_Down

Enrichment

(b)

Figure 7: The DEmiRNA-small molecular drug network involved in the NSPC-induced EC alterations (a) and EC-induced NSPC alterations (b). Red squares represent upregulated miRNAs, green squares indicate downregulated miRNAs, and yellow diamonds indicate miRNArelated small molecular drugs.

example, the addition of ECs into NSPCs was noted to cause the upregulation of the gene Chuk (Component of Inhibitor of Nuclear Factor Kappa B Kinase) in NSPCs. The encoded protein of Chuk is a component of a cytokine-activated protein complex that acts as an inhibitor of the transcription factor NF- $\kappa \mathrm{B}$ complex [51]. The Chuk gene overexpressed in 
TABLE 13: The 16 small molecular drugs that were overlapped between NSPC-induced EC genetic alteration and EC-induced NSPC genetic alteration.

\begin{tabular}{lcc}
\hline Small molecular drugs & Enrichment in the NSPC-induced EC alteration & Enrichment in the EC-induced NSPC alteration \\
\hline 2-Aminobenzenesulfonamide & 0.629 & -0.682 \\
Adiphenine & 0.748 & -0.693 \\
Deptropine & -0.677 & -0.672 \\
Diphenhydramine & 0.67 & -0.573 \\
Felbinac & 0.719 & -0.697 \\
Fludrocortisone & 0.503 & -0.496 \\
Fluphenazine & -0.357 & -0.378 \\
Ikarugamycin & 0.811 & -0.838 \\
Lycorine & 0.633 & 0.776 \\
Milrinone & -0.779 & 0.73 \\
PHA-00745360 & 0.567 & -0.514 \\
Podophyllotoxin & 0.661 & -0.933 \\
Quinostatin & -0.992 & -0.954 \\
Resveratrol & -0.479 & -0.504 \\
Thiamazole & 0.726 & 0.543 \\
Triflupromazine & 0.694 & -0.71 \\
\hline
\end{tabular}

NSPCs might be a crosstalk gene in promoting interactions between NSPCs and ECs, based on the function of the Chuk-targeted NF- $\kappa \mathrm{B}$ pathway in regulating neurogenesis [52] and angiogenesis [53]. The activation of the NF- $\kappa \mathrm{B}$ pathway is known to be involved in angiogenesis by increasing the proliferating potential of vascular ECs and increasing the expression levels of vascular growth factors [53]. In addition, the regulatory function of the NF- $\kappa \mathrm{B}$ pathway in neurogenesis is reflected in its role in initiating the early differentiation of NSPCs [52]. In summary, previous evidence supports the notion that miR-23a plays a role in promoting the interactions between ECs and NSPCs by targeting genes and pathways relevant to angiogenesis and neurogenesis.

The research value of the current research should be highlighted by the comparison between the previously published paper based on the same dataset GSE29759 [10] and the results obtained by the current research. In the previously published paper [10], the authors performed differential expression analysis and have listed the up- and downregulated DEmiRNAs which were differentially expressed in NSPC-induced EC alteration and EC-induced NSPC alteration, respectively. However, further deep investigation about the crosstalk miRNAs linking the interaction between NSPCs and ECs is lacking. In order to remedy this research gap, the present research made full use of this valuable dataset with reasonable study design by performing a series of comprehensive bioinformatics analysis and finally obtained the main results showing the crosstalk role of three miRNAs (miR-210, miR-230a, and miR-23b) in the reciprocal interaction between ECs and NSPCs. Apart from this main finding, the present research also identified that some other genetic biomarkers (i.e., six genes (i.e., MMP14, TIMP3, LOXL1, CCK, SMAD6, and HSPA2) and three pathways (i.e., Akt,
ERK1/2, and BMPs)) play critical roles in the interaction of ECs and NSPCs. And also, some target drugs (six small molecular drugs (i.e., deptropine, fluphenazine, lycorine, quinostatin, resveratrol, and thiamazole) and seven environmental chemical compounds (i.e., folic acid, dexamethasone, choline, doxorubicin, thalidomide, bisphenol $\mathrm{A}$, and titanium dioxide)) were identified to influence angiogenesis and neurogenesis by regulating the expression of DEmiRNAs. These results obtained by the current research not only fully explained the underlying information of the dataset GSE29759 but also facilitated neurology researchers to have a better understanding of the genetic and epigenetic mechanisms involved in the interaction between ECs and NSPCs.

It is important to acknowledge the limitation of this research. The first limitation is lacking experimental validation using quantitative real-time polymerase chain reaction (qRT-PCR) to verify the dysregulation of biomarkers identified during the reciprocal alterations between ECs and NPSCs; however, investigating the expression patterns and detailed functions of these biomarkers (especially 3 miRNAs (miR-210, miR-230a, and miR-23b)) as the crosstalks between ECs and NPSCs could be a separate research study and within the research plan of our team. The second limitation is only one dataset (GSE29759) related to this research topic was included, which means the sample size that was analyzed was comparatively limited. This indicates that the future RNA-sequencing study could follow the same study design; if so, more datasets could be combined and analyzed together, which could increase the prediction accuracy of the results identified by bioinformatics analyses. The third limitation is the dataset (GSE29759) only examined the messenger RNA and miRNA expression profiles. Since noncoding RNAs contain miRNAs, long noncoding RNAs (lncRNAs), 


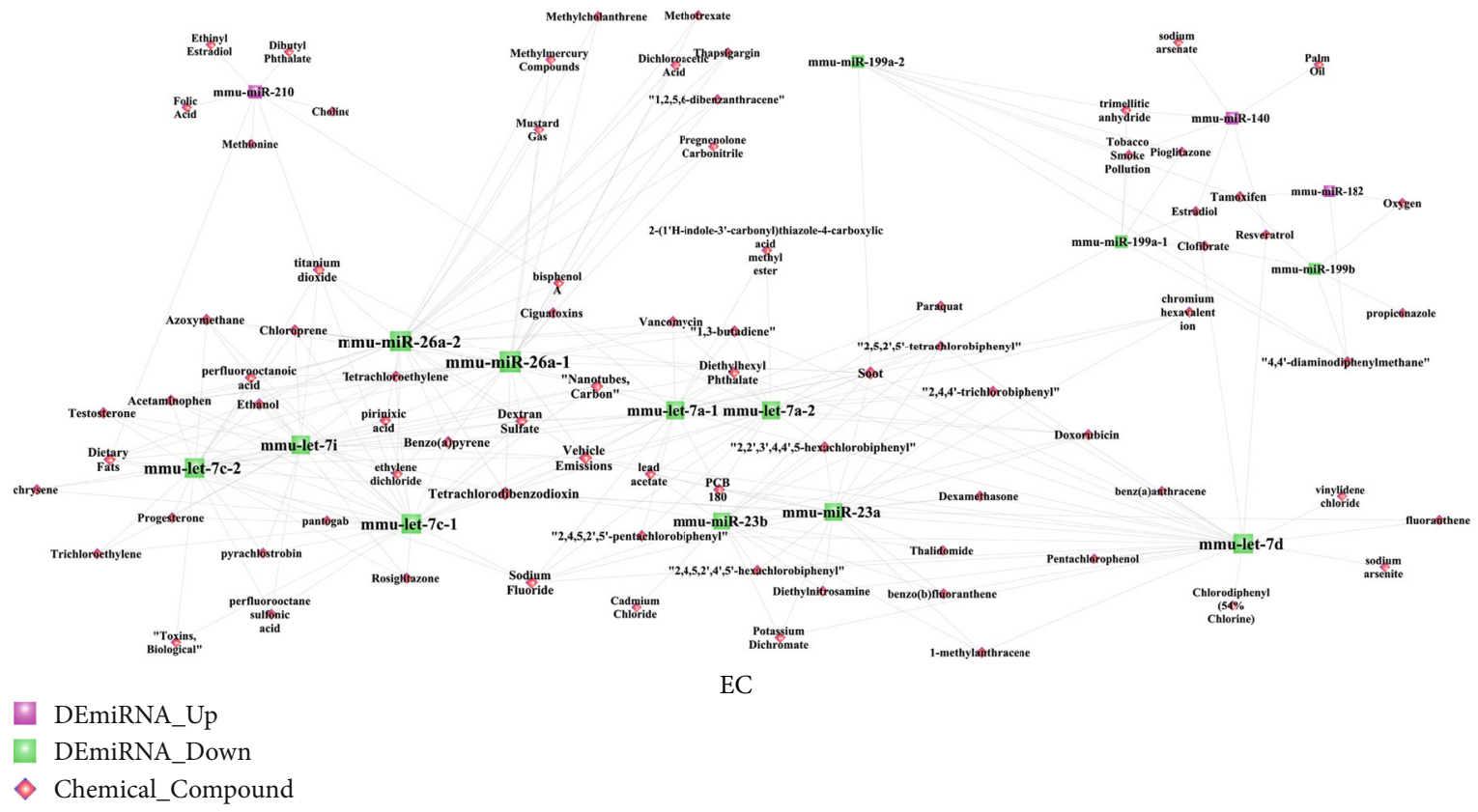

Chemical_Compound

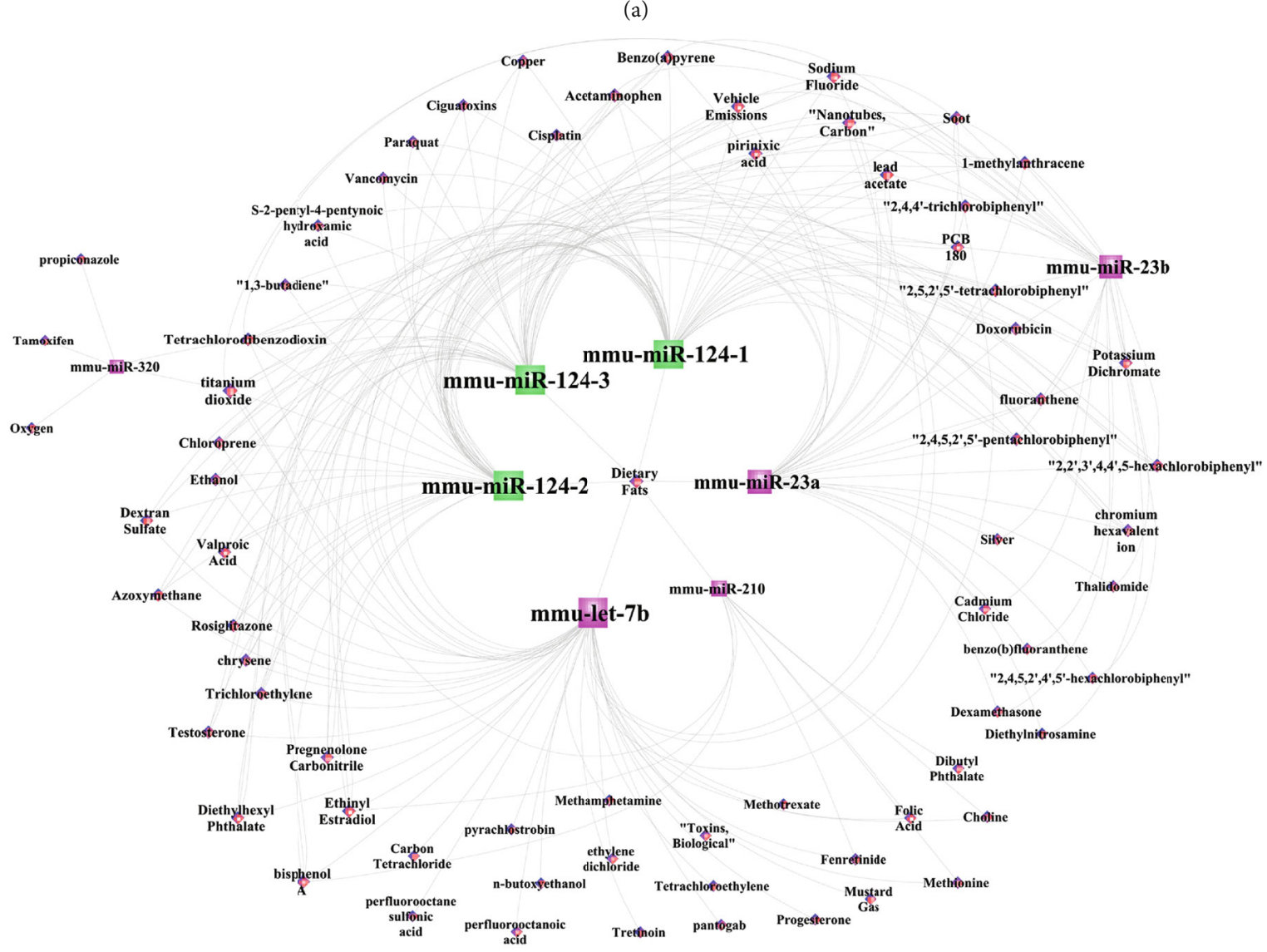

DEmiRNA_Up

DEmiRNA_Down

Chemical_Compound

(b)

FIGURE 8: The DEmiRNA-chemical compound network involved in the NSPC-induced EC alterations (a) and EC-induced NSPC alterations (b). Red squares represent upregulated miRNAs, green squares indicate downregulated miRNAs, and pink diamonds indicate miRNA-related chemical compounds. 
TABLE 14: The scores of the crosstalk miRNA interaction pairs under five conditions including the target genes, small molecular drugs, chemical compounds, signaling pathways, and biological processes. These crosstalk miRNAs link the EC-induced NSPC alteration process and NSPC-induced EC alteration process. The scores were calculated by the "Meet/Min" formula.

\begin{tabular}{|c|c|c|c|c|c|c|}
\hline $\begin{array}{l}\text { miRNAs involved in the NSPC- } \\
\text { induced EC alteration }\end{array}$ & $\begin{array}{l}\text { miRNAs involved in the EC-induced } \\
\text { NSPC alteration }\end{array}$ & $\begin{array}{c}\text { Score_ } \\
\text { target }\end{array}$ & $\begin{array}{l}\text { Score__ } \\
\text { molecular }\end{array}$ & $\begin{array}{l}\text { Score__ } \\
\text { chemical }\end{array}$ & $\begin{array}{l}\text { Score_- } \\
\text { pathway }\end{array}$ & $\begin{array}{l}\text { Score } \\
\text { bp }\end{array}$ \\
\hline mmu-miR-210 & mmu-miR-210 & 1 & 0.166667 & 1 & 1 & 1 \\
\hline mmu-miR-23b & mmu-miR-23b & 1 & 0.075 & 1 & 1 & 1 \\
\hline mmu-miR-23b & mmu-miR-23a & 0.966903 & 0.022222 & 1 & 0.946667 & 0.931284 \\
\hline mmu-miR-23a & mmu-miR-23b & 0.966903 & 0.05 & 0.95 & 0.946667 & 0.931284 \\
\hline mmu-let-7d & mmu-let-7b & 0.834665 & 0.021739 & 0.2 & 1 & 0.862256 \\
\hline mmu-miR-26a-1 & mmu-miR-23a & 0.393215 & 0.05 & 0.321429 & 0.936709 & 0.614696 \\
\hline mmu-miR-26a-2 & mmu-miR-23a & 0.393215 & 0.05 & 0.321429 & 0.936709 & 0.614696 \\
\hline mmu-miR-26a-1 & mmu-miR-23b & 0.389283 & 0.025 & 0.307692 & 0.946667 & 0.615335 \\
\hline mmu-miR-26a-2 & mmu-miR-23b & 0.389283 & 0.025 & 0.307692 & 0.946667 & 0.615335 \\
\hline mmu-miR-26a-1 & mmu-miR-210 & 0.344017 & 0.027778 & 0.333333 & 0.8 & 0.689956 \\
\hline mmu-miR-26a-2 & mmu-miR-210 & 0.344017 & 0.027778 & 0.333333 & 0.8 & 0.689956 \\
\hline mmu-miR-26a-1 & mmu-miR-320 & 0.337681 & 0.05 & 0.4 & 0.885714 & 0.708661 \\
\hline mmu-miR-26a-2 & mmu-miR-320 & 0.337681 & 0.05 & 0.4 & 0.885714 & 0.708661 \\
\hline mmu-miR-23a & mmu-let-7b & 0.312259 & 0.021739 & 0.25 & 0.772152 & 0.595539 \\
\hline mmu-let-7i & mmu-miR-23b & 0.3026 & 0.074074 & 0.318182 & 0.693333 & 0.592737 \\
\hline mmu-let-7i & mmu-miR-23a & 0.302236 & 0.074074 & 0.363636 & 0.708861 & 0.590143 \\
\hline mmu-miR-199a-1 & mmu-let-7b & 0.299166 & 0.022222 & 0.142857 & 0.728395 & 0.65798 \\
\hline mmu-miR-199a-2 & mmu-let-7b & 0.299166 & 0.022222 & 0.142857 & 0.728395 & 0.65798 \\
\hline mmu-let-7c-1 & mmu-miR-23b & 0.29866 & 0.071429 & 0.32 & 0.72 & 0.587937 \\
\hline mmu-let-7c-2 & mmu-miR-23b & 0.29866 & 0.071429 & 0.32 & 0.72 & 0.587937 \\
\hline mmu-let-7i & mmu-miR-210 & 0.297009 & 0.037037 & 0.222222 & 0.8 & 0.609898 \\
\hline mmu-miR-210 & mmu-let-7b & 0.292735 & 0.073171 & 0.875 & 0.8 & 0.63901 \\
\hline mmu-let-7c-1 & mmu-miR-124-1 & 0.285561 & 0.035714 & 0.72 & 0.709302 & 0.623492 \\
\hline mmu-let-7c-1 & mmu-miR-124-2 & 0.285561 & 0.035714 & 0.72 & 0.709302 & 0.623492 \\
\hline mmu-let-7c-1 & mmu-miR-124-3 & 0.285561 & 0.035714 & 0.72 & 0.709302 & 0.623492 \\
\hline mmu-let-7c-2 & mmu-miR-124-1 & 0.285561 & 0.035714 & 0.72 & 0.709302 & 0.623492 \\
\hline mmu-let-7c-2 & mmu-miR-124-2 & 0.285561 & 0.035714 & 0.72 & 0.709302 & 0.623492 \\
\hline mmu-let-7c-2 & mmu-miR-124-3 & 0.285561 & 0.035714 & 0.72 & 0.709302 & 0.623492 \\
\hline mmu-let-7a-1 & mmu-miR-23b & 0.239559 & 0.041667 & 0.619048 & 0.706667 & 0.616536 \\
\hline mmu-let-7a-2 & mmu-miR-23b & 0.239559 & 0.041667 & 0.619048 & 0.706667 & 0.616536 \\
\hline mmu-let-7a-1 & mmu-miR-23a & 0.2367 & 0.041667 & 0.666667 & 0.721519 & 0.610834 \\
\hline mmu-let-7a-2 & mmu-miR-23a & 0.2367 & 0.041667 & 0.666667 & 0.721519 & 0.610834 \\
\hline mmu-miR-23b & mmu-miR-124-1 & 0.230102 & 0.028571 & 0.666667 & 0.8 & 0.527677 \\
\hline mmu-miR-23b & mmu-miR-124-2 & 0.230102 & 0.028571 & 0.666667 & 0.8 & 0.527677 \\
\hline mmu-miR-23b & mmu-miR-124-3 & 0.230102 & 0.028571 & 0.666667 & 0.8 & 0.527677 \\
\hline mmu-miR-23a & mmu-miR-124-1 & 0.229761 & 0.028571 & 0.75 & 0.759494 & 0.523207 \\
\hline mmu-miR-23a & mmu-miR-124-2 & 0.229761 & 0.028571 & 0.75 & 0.759494 & 0.523207 \\
\hline mmu-miR-23a & mmu-miR-124-3 & 0.229761 & 0.028571 & 0.75 & 0.759494 & 0.523207 \\
\hline mmu-miR-23a & mmu-miR-320 & 0.226087 & 0.04 & 0.2 & 0.714286 & 0.755906 \\
\hline mmu-miR-23b & mmu-miR-320 & 0.22029 & 0.043478 & 0.2 & 0.628571 & 0.76378 \\
\hline mmu-miR-199a-1 & mmu-miR-23a & 0.212157 & 0.066667 & 0.142857 & 0.594937 & 0.619707 \\
\hline mmu-miR-199a-2 & mmu-miR-23a & 0.212157 & 0.066667 & 0.142857 & 0.594937 & 0.619707 \\
\hline mmu-miR-199a-1 & mmu-miR-23b & 0.205006 & 0.05 & 0.142857 & 0.626667 & 0.618078 \\
\hline mmu-miR-199a-2 & mmu-miR-23b & 0.205006 & 0.05 & 0.142857 & 0.626667 & 0.618078 \\
\hline mmu-miR-210 & mmu-miR-23a & 0.202991 & 0.170732 & 0.25 & 0.8 & 0.660844 \\
\hline mmu-miR-210 & mmu-miR-23b & 0.196581 & 0.075 & 0.125 & 0.8 & 0.672489 \\
\hline
\end{tabular}


TABle 14: Continued.

\begin{tabular}{|c|c|c|c|c|c|c|}
\hline $\begin{array}{l}\text { miRNAs involved in the NSPC- } \\
\text { induced EC alteration }\end{array}$ & $\begin{array}{l}\text { miRNAs involved in the EC-induced } \\
\text { NSPC alteration }\end{array}$ & $\begin{array}{c}\text { Score__ } \\
\text { target }\end{array}$ & $\begin{array}{l}\text { Score_- } \\
\text { molecular }\end{array}$ & $\begin{array}{l}\text { Score_- } \\
\text { chemical }\end{array}$ & $\begin{array}{l}\text { Score_ } \\
\text { pathway }\end{array}$ & $\begin{array}{l}\text { Score } \\
\text { bp }\end{array}$ \\
\hline mmu-let-7d & mmu-miR-23b & 0.183706 & 0.025 & 0.64 & 0.666667 & 0.675705 \\
\hline mmu-let-7d & mmu-miR-23a & 0.182109 & 0.022222 & 0.64 & 0.717949 & 0.671367 \\
\hline mmu-let-7d & mmu-miR-320 & 0.176812 & 0.057692 & 0.2 & 0.485714 & 0.531496 \\
\hline mmu-miR-199a-1 & mmu-miR-320 & 0.127536 & 0.022222 & 0.2 & 0.771429 & 0.616142 \\
\hline mmu-miR-199a-2 & mmu-miR-320 & 0.127536 & 0.022222 & 0.2 & 0.771429 & 0.616142 \\
\hline mmu-miR-182 & mmu-miR-320 & 0.115269 & 0.093023 & 0.666667 & 0.828571 & 0.555118 \\
\hline mmu-miR-199b & mmu-miR-320 & 0.109091 & 0.090909 & 0.6 & 0.714286 & 0.543307 \\
\hline mmu-miR-210 & mmu-miR-320 & 0.087607 & 0.121951 & 0.2 & 0.2 & 0.358268 \\
\hline
\end{tabular}

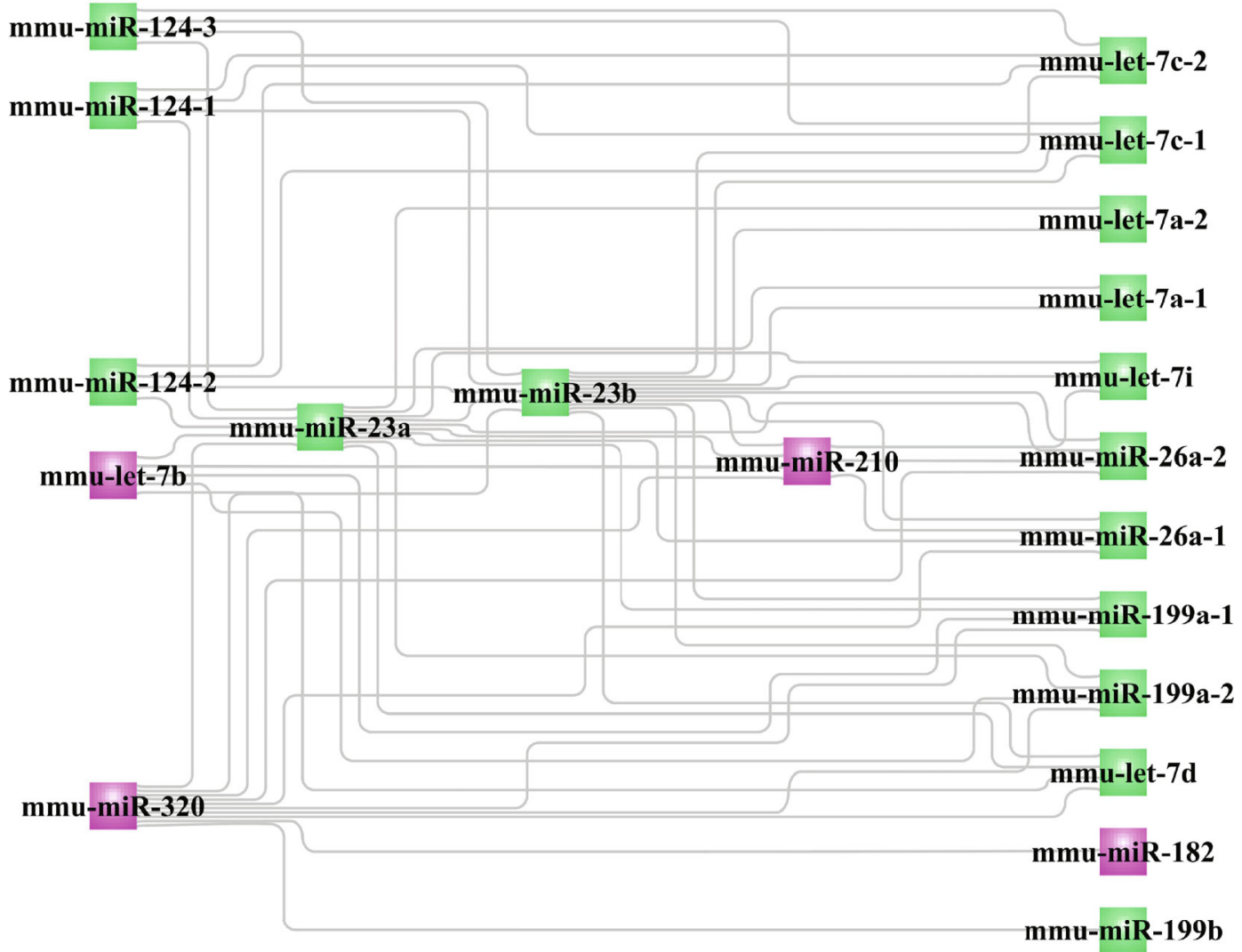

DEmiRNA_Up

DEmiRNA_Down

FIGURE 9: The crosstalk DEmiRNA interaction network involved in the link between the EC-induced NSPC alteration process and the NSPCinduced EC alteration process.

circular RNAs (circRNAs), and Piwi-interacting RNA (piRNA), the future research with the same study design could further examine the other noncoding RNA expression profile alterations that occurred during the reciprocal changes in the interactions between ECs and NPSCs. If more datasets with the design of examining the other noncoding RNA expression profiles were included in this analysis, then constructing a circRNA/lncRNA-miRNA-mRNA competitive endogenous RNA (ceRNA) interaction network will be more meaningful for investigating the genetic and epigenetic crosstalks involved in the reciprocal interactions between ECs and NPSCs.

Although limitations exist in this research, the present study also provides implications for future research. First, 
the genetic and epigenetic linkage mechanisms as well as drugs that are involved in the interactions between ECs and NSPCs could translate to novel gene/drug delivery strategies for constructing 3D neurovascular tissue. With the rapid advancements in genome-editing techniques, it may be possible to perform the gene modifications on stem cells (i.e., endothelial stem-like cells and NSPCs) specific to the crosstalk genes and miRNAs identified in this study. The small molecular drugs and chemical compounds are relevant to nanoparticle research for controlled drug delivery to exert sustained effects on the neurogenic and angiogenic processes of ECs and NSPCs. Another noteworthy issue that should be investigated is the dose and concentration of drugs and chemical compounds, especially considering their toxicity and degradation rate. In addition, several of the identified entities are candidates for future research for the purpose of experimental validation, as these have not been investigated in the context of interactions between ECs and NSPCs.

In addition, the present research has potential clinical transfer values for ischemic stroke therapy. Since the current clinical treatments (e.g., intravenous administration of tissue-type plasminogen activator, early motor training, and physical therapy) could not result in complete functional recovery in ischemic stroke patients [54], construction of a multicellular EC-NPSC-based 3D neurovascular unit model might be an alternative tissue engineering approach for regeneration of stroke-affected neuronal tissue. However, there are still no clinical trials using the biomaterial scaffolds carried with ECs and NPSCs in stroke therapy. Although tissue engineering is currently fraught with many challenges $[55,56]$, the identification of genetic and epigenetic mechanisms and drugs in facilitating the interactions between ECs and NSPCs might be potentially used for the genetic/epigenetic modification of ECs/NSPCs and drug delivery of biomaterial scaffolds and thereby enhance the effects of angiogenesis and neurogenesis. By performing these modifications, the success rate of constructing a 3D neurovascular unit model might be significantly increased. Therefore, the findings of the present research may be regarded as a preliminary step providing a theoretical basis to further research toward a promising stem cell-based stroke therapy.

\section{Conclusions}

This bioinformatics study identified 3 miRNAs (miR-210, miR-230a, and miR-23b) as crosstalk entities involved in the interaction between ECs and NSPCs. These 3 miRNAs may be relevant to the arena of stem cell modification to further promote angiogenesis and neurogenesis within the field of stem cell tissue engineering.

\section{Data Availability}

The data used to support the findings of this study are available from the corresponding author upon request.

\section{Conflicts of Interest}

The authors declare no potential conflict of interest with respect to the authorship and publication of this paper.

\section{Authors' Contributions}

Bernd Lethaus and Vuk Savkovic contributed equally as senior authors.

\section{Acknowledgments}

The first author Xin Wang received financial support from the Research Fund of Heilongjiang Provincial Education Department, Heilongjiang Province, China (Grant No. 31041180068).

\section{References}

[1] V. Muoio, P. B. Persson, and M. M. Sendeski, "The neurovascular unit - concept review," Acta Physiologica, vol. 210, no. 4, pp. 790-798, 2014.

[2] G. Potjewyd, S. Moxon, T. Wang, M. Domingos, and N. M. Hooper, "Tissue engineering 3D neurovascular units: a biomaterials and bioprinting perspective," Trends in Biotechnology, vol. 36, no. 4, pp. 457-472, 2018.

[3] O. Rajkovic, G. Potjewyd, and E. Pinteaux, "Regenerative medicine therapies for targeting neuroinflammation after stroke," Frontiers in Neurology, vol. 9, p. 734, 2018.

[4] T. Roitbak, L. Li, and L. A. Cunningham, "Neural stem/progenitor cells promote endothelial cell morphogenesis and protect endothelial cells against ischemia via HIF-1alpharegulated VEGF signaling," Journal of Cerebral Blood Flow and Metabolism, vol. 28, no. 9, pp. 1530-1542, 2008.

[5] J. M. Rosenstein, J. M. Krum, and C. Ruhrberg, "VEGF in the nervous system," Organogenesis, vol. 6, no. 2, pp. 107-114, 2014.

[6] O. A. Levada, N. V. Cherednichenko, A. V. Trailin, and A. S. Troyan, "Plasma brain-derived neurotrophic factor as a biomarker for the main types of mild neurocognitive disorders and treatment efficacy: a preliminary study," Disease Markers, vol. 2016, Article ID 4095723, 7 pages, 2016.

[7] N. Nakagomi, T. Nakagomi, S. Kubo et al., "Endothelial cells support survival, proliferation, and neuronal differentiation of transplanted adult ischemia-induced neural stem/progenitor cells after cerebral infarction," Stem Cells, vol. 27, no. 9, pp. 2185-2195, 2009.

[8] Q. Shen, S. K. Goderie, L. Jin et al., "Endothelial cells stimulate self-renewal and expand neurogenesis of neural stem cells," Science, vol. 304, no. 5675, pp. 1338-1340, 2004.

[9] M. J. Donovan, M. I. Lin, P. Wiegn et al., "Brain derived neurotrophic factor is an endothelial cell survival factor required for intramyocardial vessel stabilization," Development, vol. 127, no. 21, pp. 4531-4540, 2000.

[10] T. Roitbak, O. Bragina, J. L. Padilla, and G. G. Pickett, "The role of microRNAs in neural stem cell-supported endothelial morphogenesis," Vascular Cell, vol. 3, no. 1, p. 25, 2011.

[11] S. Zhang, L. Chen, E. J. Jung, and G. A. Calin, "Targeting microRNAs with small molecules: from dream to reality," Clinical Pharmacology and Therapeutics, vol. 87, no. 6, pp. $754-758,2010$. 
[12] X. Wu and Y. Song, "Preferential regulation of miRNA targets by environmental chemicals in the human genome," BMC Genomics, vol. 12, no. 1, p. 244, 2011.

[13] J. Reinhardt, J. Landsberg, J. L. Schmid-Burgk et al., "MAPK signaling and inflammation link melanoma phenotype switching to induction of CD73 during immunotherapy," Cancer Research, vol. 77, no. 17, pp. 4697-4709, 2017.

[14] G. Yu, L. G. Wang, Y. Han, and Q. Y. He, “clusterProfiler: an R package for comparing biological themes among gene clusters," OMICS, vol. 16, no. 5, pp. 284-287, 2012.

[15] D. Szklarczyk, A. L. Gable, D. Lyon et al., "STRING v11: protein-protein association networks with increased coverage, supporting functional discovery in genome-wide experimental datasets," Nucleic Acids Research, vol. 47, no. D1, pp. D607D613, 2019.

[16] P. Shannon, A. Markiel, O. Ozier et al., "Cytoscape: a software environment for integrated models of biomolecular interaction networks," Genome Research, vol. 13, no. 11, pp. 24982504, 2003.

[17] H. Han, J.-W. Cho, S. Lee et al., "TRRUST v2: an expanded reference database of human and mouse transcriptional regulatory interactions," Nucleic Acids Research, vol. 46, no. D1, pp. D380-D386, 2018.

[18] H.-Y. Huang, Y.-C.-D. Lin, J. Li et al., “miRTarBase 2020: updates to the experimentally validated microRNA-target interaction database," Nucleic Acids Research, vol. 48, no. D1, pp. D148-D154, 2020.

[19] F. Xiao, Z. Zuo, G. Cai, S. Kang, X. Gao, and T. Li, "miRecords: an integrated resource for microRNA-target interactions," Nucleic Acids Research, vol. 37, no. Database, pp. D105D110, 2009.

[20] I. S. Vlachos, M. D. Paraskevopoulou, D. Karagkouni et al., "DIANA-TarBase v7. 0: indexing more than half a million experimentally supported miRNA: mRNA interactions," Nucleic Acids Research, vol. 43, no. D1, pp. D153-D159, 2015.

[21] V. Agarwal, G. W. Bell, J.-W. Nam, and D. P. Bartel, "Predicting effective microRNA target sites in mammalian mRNAs," eLife, vol. 4, article e05005, 2015.

[22] J.-H. Yang, J.-H. Li, P. Shao, H. Zhou, Y.-Q. Chen, and L.H. Qu, "starBase: a database for exploring microRNA-mRNA interaction maps from Argonaute CLIP-Seq and DegradomeSeq data," Nucleic Acids Research, vol. 39, Supplement 1, pp. D202-D209, 2011.

[23] S.-D. Hsu, C.-H. Chu, A.-P. Tsou et al., "miRNAMap 2.0: genomic maps of microRNAs in metazoan genomes," Nucleic Acids Research, vol. 36, Supplement 1, pp. D165-D169, 2007.

[24] Y. Chen and X. Wang, "miRDB: an online database for prediction of functional microRNA targets," Nucleic Acids Research, vol. 48, no. D1, pp. D127-D131, 2020.

[25] H. Dweep, C. Sticht, P. Pandey, and N. Gretz, "miRWalk database: prediction of possible miRNA binding sites by "walking" the genes of three genomes," Journal of Biomedical Informatics, vol. 44, no. 5, pp. 839-847, 2011.

[26] J. Krüger and M. Rehmsmeier, "RNAhybrid: microRNA target prediction easy, fast and flexible," Nucleic Acids Research, vol. 34, Supplement 2, pp. W451-W454, 2006.

[27] J. Lamb, E. D. Crawford, D. Peck et al., "The connectivity map: using gene-expression signatures to connect small molecules, genes, and disease," Science, vol. 313, no. 5795, pp. 19291935, 2006.
[28] A. P. Davis, C. J. Grondin, R. J. Johnson et al., "The comparative toxicogenomics database: update 2019," Nucleic Acids Research, vol. 47, no. D1, pp. D948-D954, 2019.

[29] K.-I. Katayama, J. Melendez, J. M. Baumann et al., "Loss of RhoA in neural progenitor cells causes the disruption of adherens junctions and hyperproliferation," Proceedings of the National Academy of Sciences of the United States of America, vol. 108, no. 18, pp. 7607-7612, 2011.

[30] A. J. Keung, E. M. de Juan-Pardo, D. V. Schaffer, and S. Kumar, "Rho GTPases mediate the mechanosensitive lineage commitment of neural stem cells," Stem Cells, vol. 29, no. 11, pp. 18861897, 2011.

[31] G. P. van Nieuw Amerongen, P. Koolwijk, A. Versteilen, and V. W. M. van Hinsbergh, "Involvement of RhoA/Rho kinase signaling in VEGF-induced endothelial cell migration and angiogenesis in vitro," Arteriosclerosis, Thrombosis, and Vascular Biology, vol. 23, no. 2, pp. 211-217, 2003.

[32] C. A. Escudero, K. Herlitz, F. Troncoso et al., "Pro-angiogenic role of insulin: from physiology to pathology," Frontiers in Physiology, vol. 8, p. 204, 2017.

[33] A. N. Ziegler, S. W. Levison, and T. L. Wood, "Insulin and IGF receptor signalling in neural-stem-cell homeostasis," Nature Reviews Endocrinology, vol. 11, no. 3, pp. 161-170, 2015.

[34] M. C. Tate, D. A. Shear, S. W. Hoffman, D. G. Stein, D. R. Archer, and M. C. Laplaca, "Fibronectin promotes survival and migration of primary neural stem cells transplanted into the traumatically injured mouse brain," Cell Transplantation, vol. 11, no. 3, pp. 283-295, 2002.

[35] V. B. S. Kumar, R. I. Viji, M. S. Kiran, and P. R. Sudhakaran, "Angiogenic response of endothelial cells to fibronectin," Advances in Experimental Medicine and Biology, vol. 749, pp. 131-151, 2012.

[36] S. H. Koh and E. H. Lo, "The role of the PI3K pathway in the regeneration of the damaged brain by neural stem cells after cerebral infarction," Journal of Clinical Neurology, vol. 11, no. 4, pp. 297-304, 2015.

[37] J. Karar and A. Maity, "PI3K/AKT/mTOR pathway in angiogenesis," Frontiers in Molecular Neuroscience, vol. 4, p. 51, 2011.

[38] K. Ebnet, M. Aurrand-Lions, A. Kuhn et al., "The junctional adhesion molecule (JAM) family members JAM-2 and JAM3 associate with the cell polarity protein PAR-3: a possible role for JAMs in endothelial cell polarity," Journal of Cell Science, vol. 116, no. 19, pp. 3879-3891, 2003.

[39] T. Hikita, F. Mirzapourshafiyi, P. Barbacena et al., "PAR-3 controls endothelial planar polarity and vascular inflammation under laminar flow," EMBO Reports, vol. 19, no. 9, 2018.

[40] Y. Arai and E. Taverna, "Neural progenitor cell polarity and cortical development," Frontiers in Cellular Neuroscience, vol. 11, p. 384, 2017.

[41] R. J. Bodnar, "Chemokine regulation of angiogenesis during wound healing," Advances in Wound Care, vol. 4, no. 11, pp. 641-650, 2015.

[42] M. D. Krathwohl and J. L. Kaiser, "Chemokines promote quiescence and survival of human neural progenitor cells," Stem Cells, vol. 22, no. 1, pp. 109-118, 2004.

[43] A. Belmadani, P. B. Tran, D. Ren, and R. J. Miller, "Chemokines regulate the migration of neural progenitors to sites of neuroinflammation," The Journal of Neuroscience, vol. 26, no. 12, pp. 3182-3191, 2006. 
[44] K. Hida, N. Maishi, K. Akiyama et al., "Tumor endothelial cells with high aldehyde dehydrogenase activity show drug resistance," Cancer Science, vol. 108, no. 11, pp. 2195-2203, 2017.

[45] G. Vassalli, "Aldehyde dehydrogenases: not just markers, but functional regulators of stem cells," Stem Cells International, vol. 2019, Article ID 3904645, 15 pages, 2019.

[46] J. Takahashi, T. D. Palmer, and F. H. Gage, "Retinoic acid and neurotrophins collaborate to regulate neurogenesis in adultderived neural stem cell cultures," Journal of Neurobiology, vol. 38, no. 1, pp. 65-81, 1999.

[47] S. Pal, M. L. Iruela-Arispe, V. S. Harvey et al., "Retinoic acid selectively inhibits the vascular permeabilizing effect of VPF/VEGF, an early step in the angiogenic cascade," Microvascular Research, vol. 60, no. 2, pp. 112-120, 2000.

[48] M. Mongiat, E. Andreuzzi, G. Tarticchio, and A. Paulitti, "Extracellular matrix, a hard player in angiogenesis," International Journal of Molecular Sciences, vol. 17, no. 11, p. 1822, 2016.

[49] P. A. Guerrero and J. H. McCarty, "TGF- $\beta$ activation and signaling in angiogenesis," in Physiologic and Pathologic Angiogenesis - Signaling Mechanisms and Targeted Therapy, 2017.

[50] J. M. Dias, Z. Alekseenko, J. M. Applequist, and J. Ericson, "Tgf $\beta$ signaling regulates temporal neurogenesis and potency of neural stem cells in the CNS," Neuron, vol. 84, no. 5, pp. 927-939, 2014.

[51] Y. Xie, K. Xie, Q. Gou, and N. Chen, "I $\kappa$ B kinase $\alpha$ functions as a tumor suppressor in epithelial-derived tumors through an NF- $\kappa$ B-independent pathway (review)," Oncology Reports, vol. 34, no. 5, pp. 2225-2232, 2015.

[52] Y. Zhang, J. Liu, S. Yao et al., "Nuclear factor Kappa B signaling initiates early differentiation of neural stem cells," Stem Cells, vol. 30, no. 3, pp. 510-524, 2012.

[53] X. Sui, "Inhibition of the NF- $\kappa$ B signaling pathway on endothelial cell function and angiogenesis in mice with acute cerebral infarction," Journal of Biological Regulators and Homeostatic Agents, vol. 33, no. 2, pp. 375-384, 2019.

[54] J. Wang, W. Yang, H. Xie, Y. Song, Y. Li, and L. Wang, "Ischemic stroke and repair: current trends in research and tissue engineering treatments," Regenerative Medicine Research, vol. 2, no. 1, p. 3, 2014.

[55] M. Singh, P. K. Pandey, A. Bhasin, M. V. Padma, and S. Mohanty, "Application of stem cells in stroke: a multifactorial approach," Frontiers in Neuroscience, vol. 14, 2020.

[56] O. Y. Bang, E. H. Kim, J. M. Cha, and G. J. Moon, "Adult stem cell therapy for stroke: challenges and progress," Journal of Stroke, vol. 18, no. 3, pp. 256-266, 2016. 